\title{
Almost positive links are strongly quasipositive
}

\author{
Peter Feller ${ }^{1} \cdot$ Lukas Lewark $^{2}$ - Andrew Lobb ${ }^{3}$ \\ Received: 16 November 2020 / Revised: 4 July 2021 / Accepted: 24 November 2021 / \\ Published online: 11 January 2022 \\ (c) The Author(s) 2022
}

\begin{abstract}
We prove that any link admitting a diagram with a single negative crossing is strongly quasipositive. This answers a question of Stoimenow's in the (strong) positive. As a second main result, we give a simple and complete characterization of link diagrams with quasipositive canonical surface (the surface produced by Seifert's algorithm). As applications, we determine which prime knots up to 13 crossings are strongly quasipositive, and we confirm the following conjecture for knots that have a canonical surface realizing their genus: a knot is strongly quasipositive if and only if the Bennequin inequality is an equality.
\end{abstract}

\section{Mathematics Subject Classification 57M25}

\section{Introduction}

Notions of quasipositivity for links and surfaces were introduced and explored by Rudolph in a series of papers (cited in the text). Their study is motivated, for example,

Communicated by Thomas Schick.

Peter Feller

peter.feller@math.ch

https://people.math.ethz.ch/ pfeller/

Lukas Lewark

lukas@lewark.de

http://www.lewark.de/lukas/

Andrew Lobb

andrew.lobb@durham.ac.uk

http://www.maths.dur.ac.uk/users/andrew.lobb/

1 ETH Zurich, Rämistrasse 101, 8092 Zurich, Switzerland

2 Mathematical Institute, University of Bern, Alpeneggstr. 22, 3012 Bern, Switzerland

3 Mathematical Sciences, Durham University, Durham, UK 
by connections with complex algebraic plane curves $[6,20]$ and relationships to contact geometry [3,12].

Quasipositive links, strongly quasipositive links, and quasipositive Seifert surfaces are usually defined in terms of braids. In this paper, however, our focus lies more on geometry and less on braids, and so we omit the original definitions in favor of the following characterizations: a Seifert surface is called quasipositive if it is an incompressible subsurface of the fiber surface of a positive torus link (incompressible meaning that the map induced by inclusion on the fundamental group is injective) and strongly quasipositive links are precisely those links that arise as the boundary of a quasipositive Seifert surface. That these characterizations are equivalent to the original definitions is due to Rudolph [21]. We are not concerned with (non-strongly) quasipositive links in this text.

Quasipositive Seifert surfaces are of maximal Euler characteristic; not just among Seifert surfaces of the given link, but even among smooth slice surfaces [13,22].

\section{Main results}

Links that admit a positive diagram, in other words a diagram without negative crossings, are known as positive links. Positive links are strongly quasipositive [18,24]. Our first main result generalizes this to almost positive links-links admitting an almost positive diagram, in other words a diagram with a single negative crossing. This gives a positive answer to a question of Stoimenow [27, Question 4].

Theorem A Almost positive links are strongly quasipositive.

Note that the hypothesis cannot be weakened further (at least in the most obvious way), since links admitting diagrams with two negative crossings need not even be quasipositive (for example, the figure eight knot).

Almost positive links have been studied before they were given this name, and their similarity in many respects to positive links has been observed. For example, Cromwell showed that almost positive links have Conway polynomials with nonnegative coefficients [7]; Przytycki and Taniyama proved they have negative signature [19]; Stoimenow showed that non-trivial almost positive links are chiral and nonslice [26]; and Tagami proved that the 3-genus, 4-genus, and $s$ / 2 (for $s$ the Rasmussen invariant) of almost positive knots agree [31]. Theorem A can be seen in this context. In particular, Theorem A recovers the last result: the slice-Bennequin inequality implies that for any strongly quasipositive knot (and thus for any almost positive knot) the 3-genus, 4-genus, and all slice-torus invariants agree [14,15,22]. This also proves chirality and non-sliceness for non-trivial almost positive knots. Here, a slice-torus invariant $[14,15]$ is a homomorphism $y$ from the smooth concordance group to $\mathbb{R}$ such that for all knots $K, y(K)$ is a lower bound for the 4-genus of $K$, and for positive torus knots $K, y(K)$ is equal to the 4-genus of $K$. Examples of such $y$ include $\tau$ from knot Floer homology, and $s / 2$.

To prove Theorem A, we explicitly exhibit quasipositive Seifert surfaces for all almost positive links. For a certain type of almost positive diagram (which will later be referred to as type I), we prove that in fact the canonical surface is quasipositive (the canonical surface is that produced from the diagram by Seifert's algorithm). 
Canonical surfaces have been studied extensively; it is for example a classical result that the canonical surfaces of alternating diagrams are genus-minimizing [8,17], which generalizes to homogeneous diagrams [7], and has recently been scrutinized further $[28,29]$. In this light, our proof of Theorem A naturally begs the question: which canonical surfaces are quasipositive? The complete answer to this question forms our second main result: namely a criterion in terms of the Seifert graph, which is combinatorial and algorithmic.

Theorem B A canonical surface is quasipositive if and only if all cycles of its Seifert graph have strictly positive total weight.

Here, the Seifert graph $\Gamma(D)$ of a diagram $D$ has the Seifert circles as vertex set, and one edge between $k$ and $k^{\prime}$ for each crossing connecting the Seifert circles $k$ and $k^{\prime}$. It is a bipartite graph, possibly with multiple edges between two vertices. Its edges carry a weight of \pm 1 corresponding to the sign of the crossing. The total weight of a cycle is understood as the sum of the weights of the cycle's edges. The reader will find more details on Seifert graphs at the beginning of Sect. 1.

\section{Applications of Theorem B}

Theorem B implies a purely geometric criterion for quasipositivity of a canonical Seifert surface, which we state as the following corollary.

Corollary C Let $\Sigma$ be a Seifert surface that is isotopic to a canonical surface. Then $\Sigma$ is quasipositive if and only if every unknot contained in $\Sigma$ bounds a disk in $\Sigma$ or has negative induced framing by $\Sigma$.

Corollary $\mathrm{C}$ does not generalize to non-canonical Seifert surfaces; in fact, there exist non-quasipositive Seifert surfaces $\Sigma$ such that all incompressible annuli of $\Sigma$ are quasipositive Seifert surfaces [4].

Next we observe the following criterion for quasipositivity. This allows, see the example below, to determine the strong-quasipositivity status of all prime knots up to 13 crossings, in particular recovering the recently completed calculation [10] of the strong-quasipositivity status of prime knots up to 12 crossings. Throughout this subsection, let $y$ denote a slice-torus invariant.

Theorem D If $K$ is a knot with a canonical surface $\Sigma$ such that $y(K)=\operatorname{genus}(\Sigma)$, then $\Sigma$ is a quasipositive Seifert surface; in particular, $K$ is a strongly quasipositive knot.

Recall that the Bennequin inequality states $\frac{\operatorname{sl}(K)+1}{2} \leq g(K)$, where $\operatorname{sl}(K)$ is defined in either of the following two equivalent ways; see [5]:

$$
\begin{aligned}
& \operatorname{sl}(K):=\max \{\operatorname{sl}(T) \mid T \text { is a transverse representative of } K\} \\
& \operatorname{sl}(K):=\max \{\operatorname{writhe}(\beta)-n \mid \beta \text { is an } n \text {-braid with closure } K\} .
\end{aligned}
$$

It is a conjecture (popularized by Hedden, Etnyre and Van Horn-Morris amongst others) that the Bennequin inequality is an equality if and only if $K$ is strongly quasipositive; compare also [10]. As a consequence of Theorem D, we confirm this conjecture 
for knots with canonical genus $\tilde{g}$ (the minimum genus of a canonical surface) equal to the genus.

Corollary $\mathrm{E}$ Let $K$ be a knot with $\tilde{g}(K)=g(K)$, i.e. a knot for which the genus $g(K)$ is realized by a canonical surface $\Sigma$. The following are equivalent:

(1) $\Sigma$ is quasipositive,

(2) $K$ is strongly quasipositive,

(3) for $K$ the Bennequin-inequality is an equality, and

(4) $y(K)=g(K)$.

Note that for $K$ a fibered knot with fiber surface $\Sigma$, the conditions of (1)-(4) in Corollary E are also equivalent [12].

Applied to the canonical surface $\Sigma\left(p_{1}, \ldots, p_{2 n+1}\right)$ of genus $n$ of the $P\left(p_{1}, \ldots\right.$, $\left.p_{2 n+1}\right)$ pretzel knot with all $p_{1}, \ldots, p_{n} \in \mathbb{Z}$ odd, Theorem B immediately yields that this surface is quasipositive if and only if $p_{i}+p_{j}<0$ for all $1 \leq i<j \leq n$, recovering a result of Rudolph's [22,25]. Moreover, since $\Sigma\left(p_{1}, \ldots, p_{2 n+1}\right)$ is genus-minimizing [9, Theorem 3.2], Corollary E now implies the following.

Corollary $\mathrm{F}$ The $P\left(p_{1}, \ldots, p_{2 n+1}\right)$ pretzel knot with all $p_{1}, \ldots, p_{n}$ odd is strongly quasipositive if and only if $p_{i}+p_{j}<0$ for all $1 \leq i<j \leq n$.

Example We claim that if $K$ is a prime knot with crossing number $c(K) \leq 13$, then $K$ is strongly quasipositive if and only if $y(K)=g(K)$. The 'only if' direction holds for all knots. To show the 'if' direction, we rely on Stoimenow's calculation [30] that for all prime knots $K$ with $c(K) \leq 13$, it holds that $\tilde{g}(K)=m(K)$, where $m$ denotes Morton's lower bound [16] for $\tilde{g}$ coming from the Homflypt-polynomial. So, using Theorem $\mathrm{D}$, it is enough to show that all prime knots with $c(K) \leq 13$ satisfy $\tau(K)<g(K)$ or $\tau(K)=m(K)$. This is readily verified by a computer calculation.

Note that this criterion is algorithmic, and can be used in practice to determine the strong quasipositivity status of a given prime knot $K$ with $c(K) \leq 13$ : simply calculate $\tau(K)$ and $m(K) ; K$ is strongly quasipositive if and only if $\tau(K)=m(K)$.

We do not know whether $y(K)=g(K)$ also implies strong quasipositivity for prime knots $K$ with $c(K)=14$. For $c(K)=15$, we know it does not: the 2-twisted positive Whitehead double of the right-handed trefoil knot is a prime 15-crossing knot ( $15 \mathrm{n} 115646$ in the table) with $\tau=g=1$, which is not quasipositive since its Rasmussen invariant $s$ is 0 [11].

Remark Theorem B also provides a new proof for Baader's theorem [1] that a knot $K$ is positive if and only if it is strongly quasipositive and homogeneous.

Here, following [7], a knot is called homogeneous if it admits a homogeneous diagram $D$; and $D$ is called homogeneous if all edges within each block of the Seifert graph $\Gamma(D)$ carry the same weight; where a block $B$ of a graph $G$ is either an isolated vertex of $G$, or a maximal subgraph of $G$ with the property that $B$ is connected and $B \backslash v$ is connected for all vertices $v$ of $B$.

We leave it as an exercise in graph theory to show that a knot diagram $D$ is homogeneous if and only if all edges within each cycle of $\Gamma(D)$ carry the same weight.

The 'only if' direction of Baader's theorem is clear. For the 'if' direction, let a strongly quasipositive and homogeneous knot $K$ be given. Let $D$ be a homogeneous 
diagram of $K$ that is also reduced (has no nugatory crossings). For $\Sigma$ the canonical surface of $D$, we have $g(\Sigma)=g(K)$ [7]. So, by Corollary E, $\Sigma$ is quasipositive. We are going to show that $D$ is a positive diagram. Let an edge $e$ of $\Gamma(D)$ be given. Since the crossing corresponding to $e$ is not nugatory, $e$ is contained in a cycle $C$ of $\Gamma(D)$. Because $\Sigma$ is quasipositive, by Theorem B, the cycle $C$ has positive total weight. Therefore, $C$ must contain at least one edge with weight +1 . But as discussed above, the homogeneity of $D$ implies that all edges within $C$ carry the same weight, so in particular, $e$ has weight +1 . This concludes the proof.

\section{Approach to proofs}

In this subsection we give more details regarding the proofs of the main theorems. We distinguish two types of diagrams.

Definition We say that $D$ is of type $I$ if $D$ is positive, or if $D$ is almost positive and there is no positive crossing parallel to the unique negative crossing (in other words connecting the same pair of Seifert circles).

Definition We say that $D$ is of type II if it is almost positive, and there is a positive crossing parallel to the unique negative crossing.

We opted to include positive diagrams in type I because they behave similarly in our constructions as almost positive diagrams of type I; for example our proof of Theorem A in Sect. 1 for links with a diagram of type I recovers Rudolph's result that positive links are strongly quasipositive.

The distinction between type I and II is rather natural and has been made previously $[27,29,31]$. Stoimenow shows that each of the two types is realized by knots that do not admit diagrams of the other type; he further shows that an almost positive diagram has a minimal genus canonical surface if and only if it is of type I. We strengthen this result from 'minimal genus' to 'quasipositive'.

Indeed, we show that if $D$ is of type I, then the canonical surface $\Sigma(D)$ is quasipositive. If $D$ is of type II, we construct a quasipositive Seifert surface $\Sigma^{\prime}(D)$ with $\partial \Sigma^{\prime}(D)=\partial \Sigma(D)$ of genus one less than $\Sigma(D)$. The alert reader has spotted that the quasipositivity of $\Sigma(D)$ for $D$ of type I also follows from Theorem B. Nevertheless, we are going to supply an independent proof, which also serves as a warm-up for the other proofs.

The quasipositivity of links admitting diagrams of type II can be shown using a method due to Baader [2] as remarked by Tagami [31]. It does not seem clear, however, how this approach could be strengthened to give a proof of strong quasipositivity. For links admitting only diagrams of type I, even quasipositivity has not hitherto been established.

The proof strategy is similar for both theorems. The quasipositivity of the surface $\Sigma(D)$ or $\Sigma^{\prime}(D)$ is established by induction over some measure of the complexity of the Seifert graph $\Gamma(D)$. For the induction step, the following two facts about quasipositive Seifert surfaces are crucial:

(M) Murasugi sums of quasipositive Seifert surfaces are quasipositive [23], 
(S) Incompressible subsurfaces of quasipositive Seifert surfaces are quasipositive.

Note that $(\mathrm{S})$ is an immediate consequence of the characterization of quasipositive Seifert surfaces that we use.

\section{Outline of the paper}

The remainder of the paper contains the proofs of the main results. The proof of Theorem A is split into Proposition 1.1 for type I in Sect. 1, and Proposition 2.1 for type II in Sect. 2. Theorem B is proven in Sect. 3. Corollary C, Theorem D and Corollary E are proven in Sect. 4. Sections 1, 2, 3 and 4 can essentially be read independently.

\section{Almost positive links of type I}

The goal of this section is to prove the following.

Proposition 1.1 The canonical surface $\Sigma(D)$ of a diagram D of type I is quasipositive.

Let us start by providing details regarding the Seifert graph $\Gamma(D)$ of a diagram $D$, which was briefly defined in the introduction.

The set of edges adjacent to a vertex $k$ of $\Gamma(D)$ carries a cyclic ordering, which comes from the ordering of crossings around the Seifert circle $k$. Moreover, $k$ separates $\mathbb{R}^{2}$ into an interior and an exterior. So each edge adjacent to $k$ carries the additional information of on which side of $k$ it lies. We say two Seifert circles $k$ and $k^{\prime}$ are nested if one lies in the interior of the other.

If $D$ has no nested Seifert circles, that is the interior of every Seifert circle is empty, then shrinking every Seifert circle to a point provides a canonical embedding of $\Gamma(D)$ into $\mathbb{R}^{2}$. Thus, if $D$ has no nested Seifert circles, we shall treat $\Gamma(D)$ as a plane graph (i.e. a graph with a fixed embedding into $\mathbb{R}^{2}$ ).

Next, we will need two lemmas giving sufficient diagrammatic conditions for the canonical surface being a Murasugi sum and a Hopf plumbing, respectively.

Lemma 1.2 (cf. [7]) Let D be a non-split link diagram (i.e. D is not a disjoint union of link diagrams) and let $k$ be a Seifert circle of $D$. Let $D_{i}$ and $D_{e}$ be the link diagrams forming the closure of the interior and the exterior of $k$, respectively (so that $D_{i} \cap D_{e}=$ $k)$. Then $\Sigma(D)$ is a Murasugi sum of $\Sigma\left(D_{i}\right)$ and $\Sigma\left(D_{e}\right)$.

Lemma 1.3 Let $D$ be a link diagram with a positive crossing c between two Seifert circles $k$ and $k^{\prime}$ that are not nested. Let $D^{\prime}$ be the diagram obtained from $D$ by inserting another positive crossing $c^{\prime}$ that is parallel to $c$ and such that $c$ is the next crossing after $c^{\prime}$ with respect to both the cyclic orderings of crossings around $k$ and around $k^{\prime}$. Then $\Sigma\left(D^{\prime}\right)$ is the plumbing of $\Sigma(D)$ and a positive Hopf band.

Proof See Fig. 1.

Proof of Proposition 1.1 We prove that $\Sigma(D)$ is quasipositive by induction over the sum of the number of Seifert circles of $D$ and the number of crossings of $D$. 
(a)

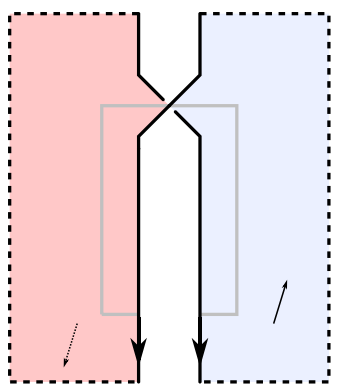

(b)

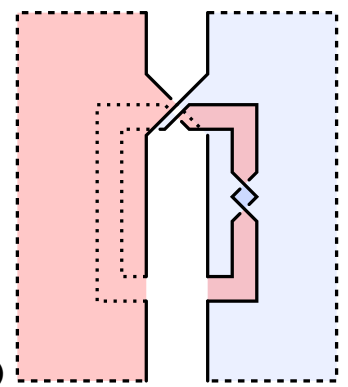

(c)

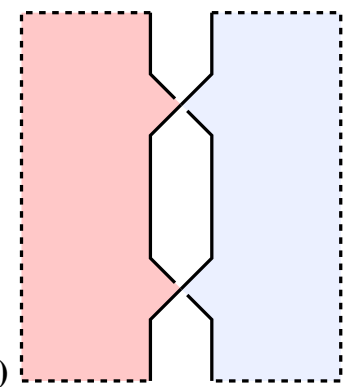

Fig. 1 Inserting a positive crossing next to another one by positive Hopf plumbing. Red and blue indicate the two sides of oriented surfaces. Dotted lines are hidden below a surface. a Two Seifert circles connected by a positive crossing. The small arrows indicate positive normal vectors of the surfaces. b Surface obtained from a by plumbing a positive Hopf band along the gray curve on the positive side of the surface. $\mathbf{c}$ This surface is isotopic to $\mathbf{b}$ (pull the Hopf band away from the crossing). Note that the central white region could contain infinity

Suppose that $D$ is a diagram with a Seifert circle that has empty exterior and nonempty interior. By 'moving infinity' we may change this to a diagram $D^{\prime}$ with no such circle and such that $\Sigma\left(D^{\prime}\right)=\Sigma(D)$. So without loss of generality we may assume that $D$ has no Seifert circle with empty exterior and non-empty interior.

Consider the following cases; we will prove below that they are exhaustive.

(1) If $D$ consists of a single Seifert circle:

The canonical surface $\Sigma(D)$ is a disk, which is quasipositive.

(2) If $D$ is split, i.e. $D=D_{1} \sqcup D_{2}$ for link diagrams $D_{1}$ and $D_{2}$ :

The surfaces $\Sigma\left(D_{i}\right)$ are quasipositive by induction, and, thus, so is $\Sigma(D)=$ $\Sigma\left(D_{1}\right) \sqcup \Sigma\left(D_{2}\right)$.

(3) If there is a nugatory crossing (i.e. an edge removing which would disconnect $\Gamma(D))$ :

Let $D^{\prime}$ denote the diagram obtained by untwisting. Then the surfaces $\Sigma(D)$ and $\Sigma\left(D^{\prime}\right)$ are isotopic and, by induction, $\Sigma\left(D^{\prime}\right)$ is quasipositive.

(4) If $D$ has a Seifert circle with non-empty interior and non-empty exterior:

If $D$ is a split diagram, we proceed as in case (2). Otherwise, the canonical Seifert surface $\Sigma(D)$ is the Murasugi sum of two canonical surfaces by Lemma 1.2. Since these two summands are quasipositive by induction, $\Sigma(D)$ is quasipositive by $(\mathrm{M})$.

(5) If there is a Seifert circle with empty interior that is adjacent to exactly two crossings, one of which is a positive crossing and the other is a negative crossing:

A Reidemeister-II-move removes that circle and the crossings adjacent to it, producing a diagram $D^{\prime}$ such that $\Sigma(D)$ and $\Sigma\left(D^{\prime}\right)$ are isotopic and $\Sigma\left(D^{\prime}\right)$ is quasipositive by induction.

(6) If a pair of non-nested Seifert circles are connected by two positive crossings that are next to each other (i.e. as in the hypothesis of Lemma 1.3):

Denote by $D^{\prime}$ the diagram obtained by deleting one of these crossings. Then $\Sigma\left(D^{\prime}\right)$ is quasipositive by induction, and $\Sigma(D)$ is a plumbing of $\Sigma\left(D^{\prime}\right)$ and a positive Hopf band by Lemma 1.3. Thus $\Sigma(D)$ is quasipositive by (M). 


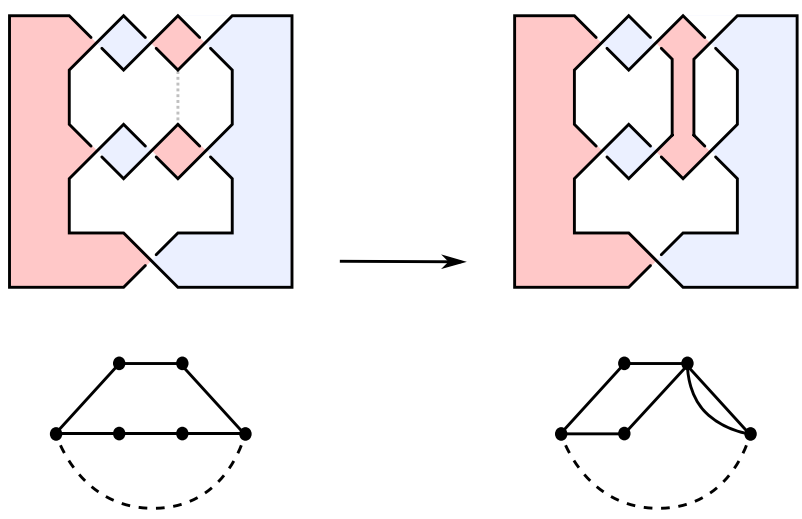

Fig. 2 Top and left: a type I diagram $D$ (the $(-3,-3,1)$-pretzel diagram) and $\Sigma(D)$. Below: its Seifert graph, with the unique edge of weight -1 drawn dashed. On the right: a diagram $D^{\prime}$ obtained from $D$ by applying (7) to the closed interval drawn gray and dotted, the surface $\Sigma\left(D^{\prime}\right)$, and the graph $\Gamma\left(D^{\prime}\right)$

(7) If there is a closed interval embedded in the plane such that

(a) its interior is disjoint from $D$,

(b) its endpoints lie on two distinct Seifert circles $k_{1}, k_{2}$,

(c) $k_{1}, k_{2}$ are oriented coherently,

(d) if $k_{1}$ and $k_{2}$ are both connected to a third circle, then both of the connecting edges are positive:

Denote by $D^{\prime}$ the diagram obtained by adding a 1 -handle along that closed interval. Because $D^{\prime}$ has one fewer Seifert circle than $D$ and $D^{\prime}$ is of type I by (7d), $\Sigma\left(D^{\prime}\right)$ is quasipositive by induction. Since $\Sigma\left(D^{\prime}\right)$ contains $\Sigma(D)$ as an incompressible subsurface, $\Sigma(D)$ is quasipositive by $(\mathrm{S})$. See Fig. 2 for an example of this case.

Let us prove that the above cases are exhaustive. For this, let us assume that (1)-(6) are not satisfied, and deduce that (7) is. Note that the exclusion of (2) and (4) implies that no Seifert circles in $D$ are nested, so its Seifert graph $\Gamma(D)$ can be seen canonically as a plane graph. Furthermore, the exclusion of (2) implies that $\Gamma(D)$ is a connected graph, the exclusion of (1) and (3) imply that all vertices of $\Gamma(D)$ have degree at least 2 , and by the exclusion of (5) vertices adjacent to a negative edge have degree at least 3 .

We distinguish two cases based on whether $\Gamma(D)$ contains a negative edge or not. In both cases, we succeed in finding an interval as in (7). See Fig. 3 for an illustration.

Case 1. Suppose there is no negative edge. Pick any edge $c$ connecting circles $k$ and $k_{1}$. Since $k$ has degree at least 2, one may walk from the edge $c$ in clockwise direction around $k$ until the next edge $c^{\prime}$, which connects $k$ to some Seifert circle $k_{2}$.

If $k_{1} \neq k_{2}$ then there is a closed interval as in (7) which connects $k_{1}$ and $k_{2}$ by following $c$ and $c^{\prime}$. Note that because there is no negative edge, (7d) is vacuously true.

If $k_{1}=k_{2}$, then walk clockwise around $k_{2}$ from where $c^{\prime}$ meets $k_{2}$ until we meet the next edge $c^{\prime \prime}$. If $c^{\prime \prime}=c$, then we are in case (6). If $c^{\prime \prime} \neq c$, note that topologically $c^{\prime \prime}$ cannot connect to $k$ since we would then have met it when walking clockwise along 

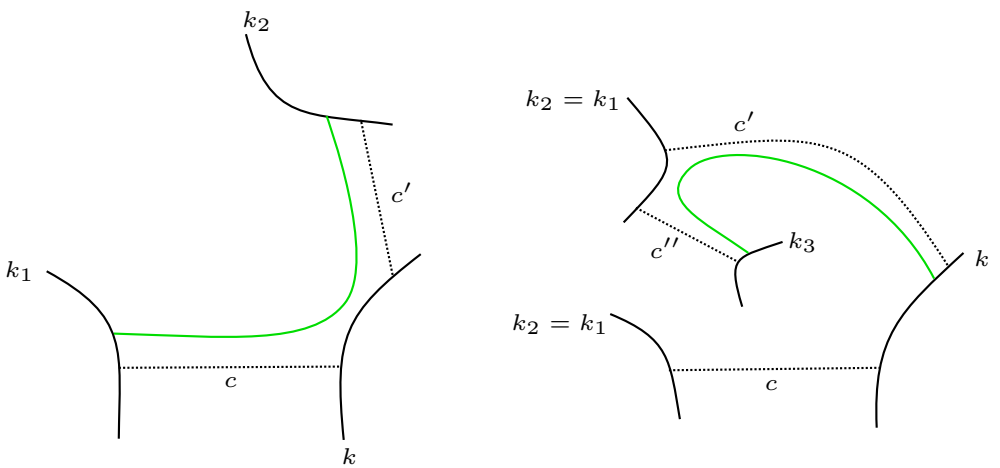

Fig. 3 How to find an interval as in (7) (drawn green)

$k$ from $c$ to $c^{\prime}$. So $c^{\prime \prime}$ connects to a Seifert circle $k_{3} \neq k$. There is then an interval connecting $k$ to $k_{3}$ by following $c^{\prime}$ and $c^{\prime \prime}$.

Case 2. Now suppose there is a negative edge connecting circles $k$ and $k_{0}$. Because $k$ has degree at least 3, one may walk from that edge in clockwise direction around $k$ until an edge $c$ connecting $k$ and some Seifert circle $k_{1}$, and still further until an edge $c^{\prime}$ connecting $k$ and some Seifert circle $k_{2}$. Because the diagram is type I, one has $k_{1} \neq k_{0}$ and $k_{2} \neq k_{0}$. Now one may proceed exactly as in the previous case to find an interval as needed for (7). We note that the intervals as constructed above also satisfy (7d) because none of $k_{1}, k_{2}$ and $k_{3}$ are adjacent to the negative edge.

\section{Almost positive links of type II}

In this section, we prove the second half of Theorem A.

Proposition 2.1 If $D$ is a link diagram of type II, then $D$ represents a strongly quasipositive link.

We first describe how to associate a Seifert surface $\Sigma^{\prime}(D)$ to such a diagram $D$, which is similar to the canonical surface but with smaller first Betti number. Afterwards we will show that $\Sigma^{\prime}(D)$ is a quasipositive Seifert surface.

Construction 1 (Generalized Seifert algorithm) Let $D$ be a diagram with exactly one negative crossing $c_{-}$. Further suppose $c_{-}$is parallel to a positive crossing $c_{+}$. If there is more than one positive crossing parallel to $c_{-}$, we fix a choice of $c_{+}$. We describe a version of Seifert's algorithm adapted to this setting that associates a Seifert surface $\Sigma^{\prime}(D)$ with the diagram $D$ as follows.

Resolve all crossings except for $c_{-}$and for $c_{+}$in the oriented manner (as in Seifert's algorithm). This produces a two-crossing diagram $D_{0}$ of an unlink $L_{0}$. The diagram $D_{0}$ consists of two twice transversely intersecting curves, which we call $s_{1}$ and $s_{2}$, and simple closed curves that are pairwise disjoint and disjoint from $s_{1}$ and $s_{2}$, which we refer to as Seifert circles. We take $s_{1}$ to be the curve that goes over. We refer to the 


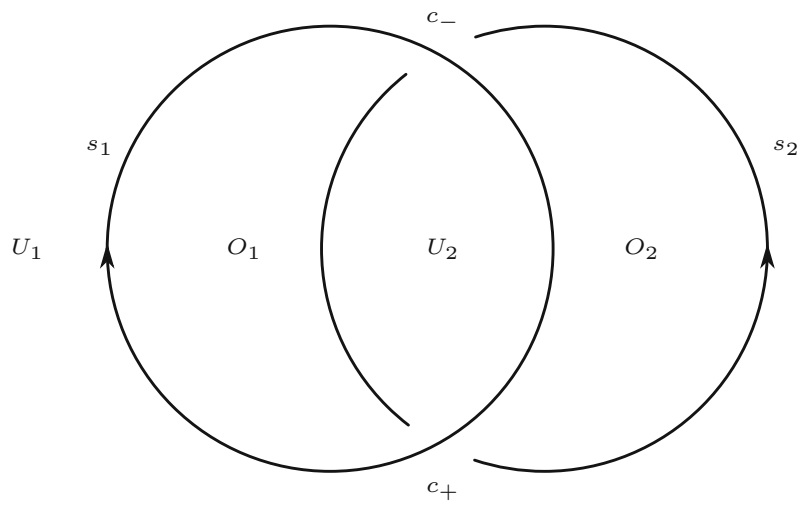

Fig. $4 s_{1}$ and $s_{2}$ cut the plane into four regions

union of the Seifert circles and $\left\{s_{1}, s_{2}\right\}$ as generalized Seifert circles. For the rest of this section, we only consider $D$ such that $s_{1}$ is oriented clockwise and $s_{2}$ is oriented counterclockwise; which, if not the case, can be achieved by 'moving infinity' without changing the associated link. See Fig. 4.

As in Seifert's algorithm, pick a disjoint union of oriented disks $d_{i}$ in $\mathbb{R}^{3}$ with constant $z$-coordinate, one for each generalized Seifert circle $k_{i}$, such that the boundary of $d_{i}$ projects to $k_{i}$ preserving orientation, and glue in a twisted ribbon for each crossing to obtain $\Sigma^{\prime}(D)$. We choose the z-coordinates for the disks as follows.

(1) The disk corresponding to $s_{1}$ has to lie above the disk corresponding to $s_{2}$. In other words (using the convention that $s_{1}$ is oriented clockwise and goes over $s_{2}$ ), the positive sides of the disks face each other.

(2) Let $k_{1}$ be a generalized Seifert circle lying wholly inside a generalized Seifert circle $k_{2}$. The disk $d_{1}$ corresponding to $k_{1}$ lies to the positive side of the disk $d_{2}$ corresponding to $k_{2}$. In other words, a positive normal to $d_{2}$ points in the direction of $d_{1}$.

Any such choice of $z$-coordinates assures that glueing in the twisted ribbons provides an embedded surface.

The choice of $z$-coordinate for disks corresponding to Seifert circles nested in $s_{1}$ and $s_{2}$ is crucial. For other disks, other choices work equally well in what is done below (save small changes in the details of the proof of Lemma 2.7).

Remark 2.2 The above generalized Seifert algorithm produces a Seifert surface out of any link diagram $D$ with two marked crossings that are parallel and of opposite sign.

When no $c_{-}$and $c_{+}$are specified, the above algorithm produces a Seifert surface from a link diagram (simply ignore (1)). However, that Seifert surface is in general not isotopic to the canonical surface, since $z$-coordinates are usually chosen differently in the usual Seifert algorithm (nested implies higher, independent of the orientations of the disks). In this section, we write $\Sigma(\cdot)$ for the Seifert surface constructed by the Seifert algorithm using the height order given in (2). 

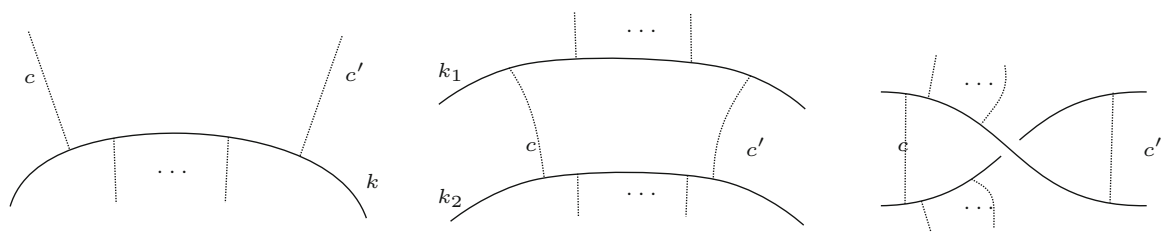

Fig. 5 Left: crossings $c$ and $c^{\prime}$ next to each other on $k$. Middle and right: crossings $c$ and $c^{\prime}$ next to each other
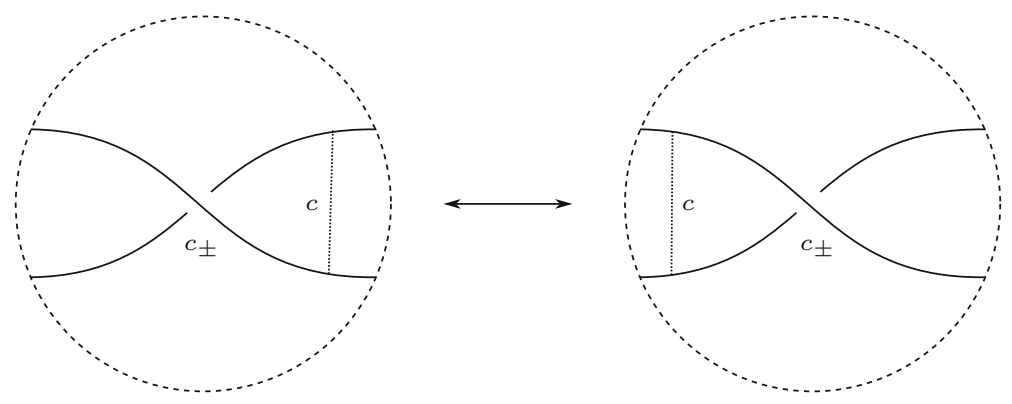

Fig. 6 Swapping the crossing $c$, which is adjacent to $s_{1}$ and $s_{2}$. Note that no other Seifert circles or crossings are present in the disk where the modification occurs

We note that the proof of Proposition 1.1 holds verbatim for the $z$-coordinate conventions in this section. Hence if $D$ is a positive diagram we already know that $\Sigma(D)$ is a quasipositive Seifert surface.

In the usual way, we specify each crossing (different from $c_{-}$and $c_{+}$) by giving an embedded closed interval $c$ (which we shall also call a crossing) in $\mathbb{R}^{2}$. The boundary points of each such crossing will lie on two different generalized Seifert circles, and each such crossing will be disjoint from $c_{-}$and $c_{+}$, with its interior disjoint from all generalized Seifert circles. See Fig. 7 for examples.

Definition 2.3 We give a few notions which we shall refer to throughout the remainder of this section.

- From here on, unless otherwise stated, a crossing refers to a crossing of $D$ that is neither $c_{+}$nor $c_{-}$.

- A crossing is said to be adjacent to the generalized Seifert circles on which its endpoints lie.

- Two crossings $c$ and $c^{\prime}$ are said to be next to each other on a generalized Seifert circle $k$, if they are both adjacent to $k$, they both lie to the same side of $k$, and there is a closed subinterval $I$ of $k$ with endpoints on $c$ and $c^{\prime}$ such that $I$ does not contain $c_{-}$or $c_{+}$and there are no crossings with endpoints on $I$ that lie to the same side of $k$ as $c$ and $c^{\prime}$. See Fig. 5(left).

- Two crossings $c$ and $c^{\prime}$ are said to be next to each other, if they are both adjacent to the same two generalized Seifert circles $k_{1}$ and $k_{2}$, they are next to each other on both $k_{i}$, witnessed by intervals $I_{i}$, such that the union $S=c \cup c^{\prime} \cup I_{1} \cup I_{2}$ has 
the property that one of the two components of $\mathbb{R}^{2} \backslash S$ contains no generalized Seifert circles and no crossings. See Fig. 5(middle).

- We also say $c$ and $c^{\prime}$ are next to each other if they are next to each other after swapping one of them over $c_{-}$or $c_{+}$; see Fig. 5(right).

- Here swapping a crossing c over $c_{-}$or over $c_{+}$is the operation on diagrams defined by a modification of a diagram in a disk as described in Fig. 6.

- The union of $s_{1}$ and $s_{2}$ separates $\mathbb{R}^{2}$ into four regions. Two of these have inconsistently oriented boundaries induced from the orientations of $s_{1}$ and $s_{2}$. We denote the unbounded region $U_{1}$ and the other $U_{2}$. We denote the remaining two regions by $O_{1}$ and $O_{2}$, where $O_{i}$ is the region contained inside $s_{i}$. See Fig. 4.

We note that if the diagram $D^{\prime}$ arises from $D$ by swapping a crossing, then $\Sigma^{\prime}\left(D^{\prime}\right)$ and $\Sigma^{\prime}(D)$ are isotopic Seifert surfaces.

Proof of Proposition 2.1 As in the proof of Proposition 1.1, we shall proceed by induction on the sum of the number of Seifert circles and the number of crossings and consider a list of cases. That these cases are exhaustive is the content of Lemma 2.5 below. We shall refer back to the proof of Proposition 1.1 for how to proceed with some of these cases.

(1') If $D$ is one of the 8 diagrams indicated in Fig. 7 or a diagram obtained from one of them by deleting Seifert circles or crossings:

The Seifert surface $\Sigma^{\prime}(D)$ is a quasipositive Seifert surface, as demonstrated in Lemma 2.6.

(2') If $D$ is split:

Proceed as in (2) (using $\Sigma^{\prime}(\cdot)$ rather than $\Sigma(\cdot)$ for the part of the diagram that contains the $s_{i}$ ). Explicitly, writing the diagram $D$ as $D_{-} \sqcup D_{+}$, where $D_{-}$contains $c_{-}$(and thus also $c_{+}$), we have that $\Sigma^{\prime}(D)=\Sigma^{\prime}\left(D_{-}\right) \sqcup \Sigma\left(D_{+}\right)$is quasipositive, since $\Sigma^{\prime}\left(D_{-}\right)$is quasipositive by induction, and $\Sigma\left(D_{+}\right)$is quasipositive because $D_{+}$is positive.

(3') If there is a nugatory crossing:

Proceed as in (3).

(4') If a Seifert circle has non-empty interior and exterior:

Proceed as in (4).

(5') If two generalized Seifert circles are connected by two crossings that are next to each other:

By Lemma 2.7 (analog of Lemma 1.3 provided at the end of this section), we obtain a diagram $D^{\prime}$ by removing one of the two crossings such that $\Sigma^{\prime}(D)$ is quasipositive if and only if $\Sigma^{\prime}\left(D^{\prime}\right)$ is quasipositive. However, by induction, $\Sigma^{\prime}\left(D^{\prime}\right)$ is a quasipositive Seifert surface.

(6') If there is a closed interval embedded in $\mathbb{R}^{2}$ such that

(a) its interior is disjoint from $D$,

(b) one of its endpoints lies on a Seifert circle $k_{1}$ and the other endpoint lies on a generalized Seifert circle $k_{2}$, and

(c) $k_{1}, k_{2}$ are oriented coherently:

Denote by $D^{\prime}$ the diagram obtained by adding a 1-handle along that closed interval. Because $D^{\prime}$ has one fewer Seifert circle than $D, \Sigma^{\prime}\left(D^{\prime}\right)$ is quasipositive by 

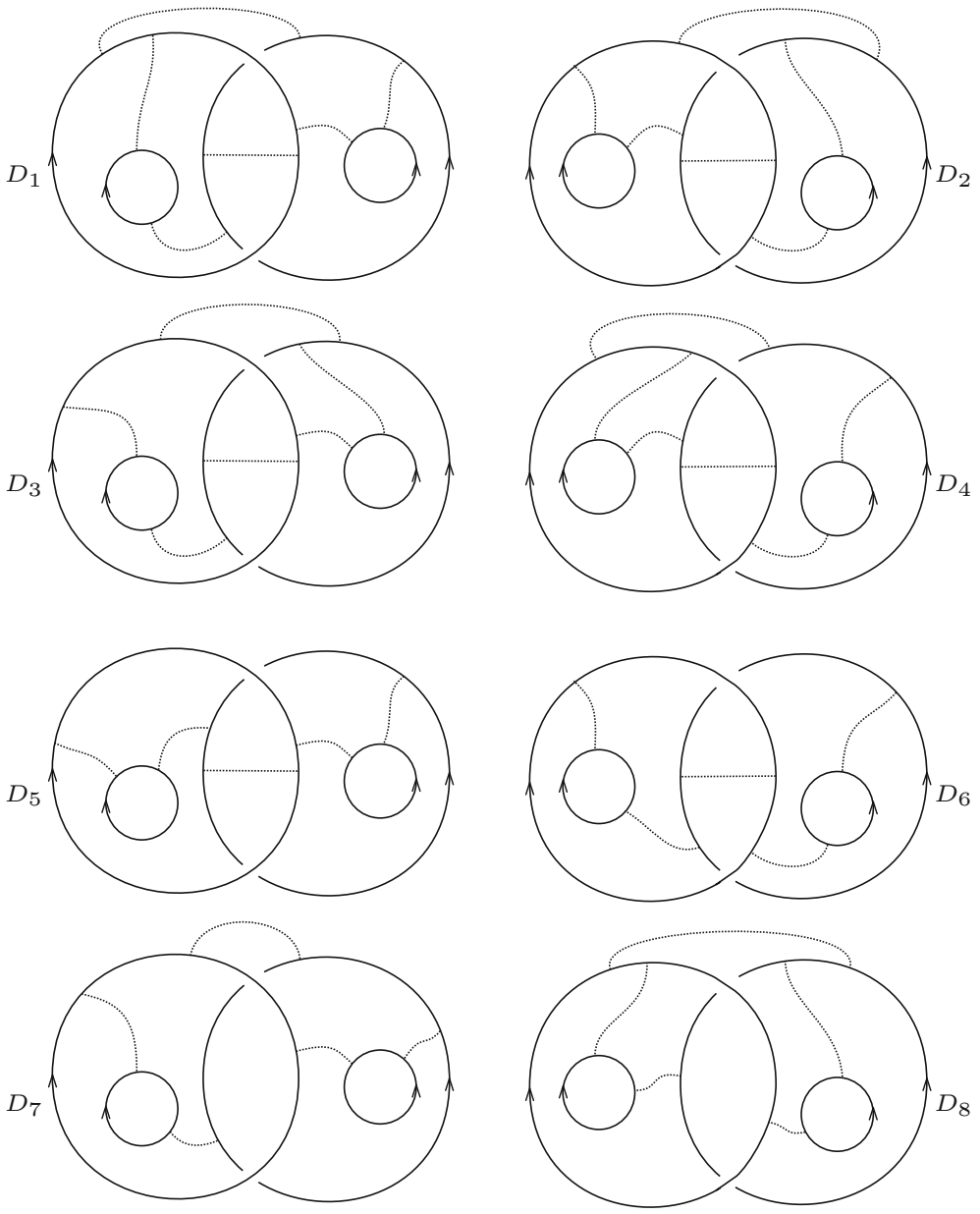

Fig. 7 Diagrams to which (2')-(6') do not apply

induction. And since $\Sigma^{\prime}\left(D^{\prime}\right)$ contains $\Sigma^{\prime}(D)$ as an incompressible subsurface, $\Sigma^{\prime}(D)$ is quasipositive by $(\mathrm{S})$.

Let us first establish that the above cases are exhaustive.

Lemma 2.4 If the conditions of none of (2')-(6') are satisfied, then

(i) the regions $U_{1}$ and $U_{2}$ contain no Seifert circles,

(ii) $\mathrm{O}_{1}$ and $\mathrm{O}_{2}$ each contain at most one Seifert circle,

(iii) each of the Seifert circles has exactly 2 positive crossings adjacent to it,

(iv) in each $U_{i}$ there is at most one crossing between $s_{1}$ and $s_{2}$.

We postpone the proof of Lemma 2.4 and apply it to prove the following.

Lemma 2.5 If D is a diagram such that (2')-(6') do not apply, then (1') applies to D. 
Fig. $8 k$ is $s_{1}$ or $s_{2}$ and $c$ is next to one of the two intersection points of $s_{1}$ and $s_{2}$ (bottom)

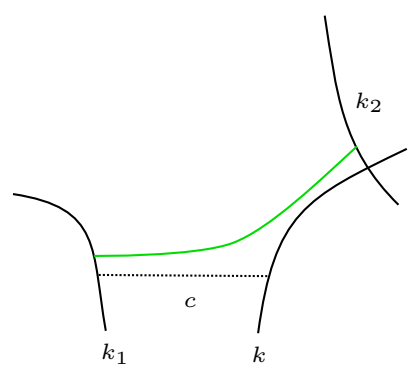

Proof By Lemma 2.4, it suffices to consider diagrams satisfying i)-iv).

First we consider the case where $D$ has a crossing in both $U_{1}$ and $U_{2}$ and $O_{1}$ and $\mathrm{O}_{2}$ each contain a Seifert circle. Once one has fixed the endpoints of the crossings in $U_{1}$ and $U_{2}$, there are four possibilities for how the two crossings adjacent to the unique Seifert circle in $O_{1}$ can lie without being next to each other (as otherwise (5') applies to $D$ ). Similarly, there are four possibilities for how the two crossings adjacent to the Seifert circle in $\mathrm{O}_{2}$ can lie without being next to each other.

Thus in this case there are 16 diagrams satisfying i)-iv). However, in 12 of these diagrams there is a crossings in $U_{1}$ that is next to a crossing of $U_{2}$ (using the notion of next to each other that uses swapping). Hence for these 12 diagrams (5') applies. The four remaining diagrams, which we denote by $D_{1}, D_{2}, D_{3}$, and $D_{4}$, are indicated in Fig. 7.

Next we consider the case where $D$ has a crossing in exactly one of $U_{1}$ or $U_{2}$, and two Seifert circles. There are eight such diagrams satisfying i)-iv). Four of these arise by deleting a crossing in one of the diagrams $D_{1}, D_{2}, D_{3}$, and $D_{4}$. The other four, denoted by $D_{5}, D_{6}, D_{7}$, and $D_{8}$, are indicated in Fig. 7 .

Finally, it is easy to see that any case not yet considered is obtained from at least one of the $D_{i}$ by deleting Seifert circles or crossings.

Proof of Lemma 2.4 i) Assume towards a contradiction that there is at least one Seifert circle, say $k_{1}$, in $U_{i}$. There is a crossing $c$ connecting $k_{1}$ to a different generalized Seifert circle $k$. We distinguish two cases.

Case 1: Assume that on $k$ there is a crossing $c^{\prime}$ next to $c$. Let $k_{2}$ be the generalized Seifert circle adjacent to $c^{\prime}$ that is not $k$; see Fig. 3 .

If $k_{1} \neq k_{2}$, then (6') applies (see green interval in Fig. 3(left)), hence we obtain a contradiction. Thus we have that $k_{1}=k_{2}$. The crossings $c^{\prime}$ and $c$ (compare Fig. 3) are next to each other on $k$, which implies that there must be another crossing $c^{\prime \prime}$ adjacent to $k_{2}$ that is between $c$ and $c^{\prime}$, since otherwise the crossings $c$ and $c^{\prime}$ are next to each other (this uses that $D$ is not split). Let $k_{3}$ be the other Seifert circle adjacent to $c^{\prime \prime}$. Now (6') applies (see green interval in Fig. 3 (right)), hence we obtain a contradiction.

Case 2: Assume that on $k$ there is no crossing next to $c$. This implies that $k$ is $s_{1}$ or $s_{2}$ (if $k$ were a Seifert circle, having no crossing next to $c$ on $k$ would imply that $c$ is the only crossing on one side of $k$, thus $c$ would be nugatory).

Thus, (6') applies (see green interval in Fig. 8), contradiction. 
ii) Assume towards a contradiction that there are at least two Seifert circles in $O_{1}$ (without loss of generality).

Case 1: Assume that inside $O_{1}$ there are two distinct crossings $c, c^{\prime}$ adjacent to $s_{i}$ for some $i$. Calling the Seifert circle $k_{1}$ that is also adjacent to $c$, we can now argue verbatim as in Case 1 of i) above.

Case 2: Assume that inside $O_{1}$ there is at most one crossing adjacent to $s_{1}$ and at most one crossing adjacent to $s_{2}$. Then, to avoid nugatory crossings, we know that there is exactly one crossing $c_{1}$ adjacent to $s_{1}$ and exactly one crossing $c_{2}$ adjacent to $s_{2}$.

Call the Seifert circle $k_{1}$ that is also adjacent to $c_{1}$. If $k_{1}$ is adjacent to no other crossings then $c_{1}$ is nugatory. If $k_{1}$ is adjacent to $c_{1}$, to $c_{2}$, and to no other crossing then either the diagram is disconnected, $k_{1}$ has non-empty interior, or there is no other Seifert circle in $O_{1}$. If $k_{1}$ is adjacent to some crossing different from $c_{1}$ and $c_{2}$, then pick $c \neq c_{2}$ to be a crossing next to $c_{1}$ on $k_{1}$. Then there is an arc connecting $s_{1}$ to the other Seifert circle adjacent to $c$.

iii) Let $i \in\{1,2\}$. Let $k$ be a Seifert circle in $O_{i}$. All crossings adjacent to $k$ are adjacent to $s_{1}$ or $s_{2}$ since there are no other Seifert circles in $O_{i}$ by ii). If there are at least three crossings, then two of them are adjacent to the same $s_{j}$ and are next to each other since there are no other Seifert circles in $O_{i}$ by ii).

iv) Let $i \in\{1,2\}$. If there are two or more crossings in $U_{i}$, then two of them are next to each other since there are no Seifert circles in $U_{i}$ by i).

It remains to show that for the diagrams $D_{i}$ for $i \in\{1, \ldots, 8\}$ given in Fig. 7, $\Sigma^{\prime}\left(D_{i}\right)$ is a quasipositive surface. This implies that $\Sigma^{\prime}(D)$ is a quasipositive Seifert surface for any diagram $D$ obtained from some $D_{i}$ by deleting crossings or Seifert circles. (Because in this case $\Sigma^{\prime}(D)$ is an incompressible subsurface of $\Sigma^{\prime}\left(D_{i}\right)$ and thus a quasipositive Seifert surface by $(\mathrm{S})$.)

Lemma 2.6 The Seifert surface $\Sigma^{\prime}\left(D_{i}\right)$ is quasipositive for all $i \in\{1, \ldots, 8\}$.

Proof We discuss each of the surfaces $\Sigma^{\prime}\left(D_{i}\right)$ in turn.

- $\mathbf{6}^{\prime}\left(\mathbf{D}_{1}\right)$. We write $L_{1}$ for the boundary of $\Sigma^{\prime}\left(D_{1}\right)$. Note that $L_{1}$ has two components and further note that $\Sigma^{\prime}\left(D_{1}\right)$ has Euler characteristic $\chi\left(\Sigma^{\prime}\left(D_{1}\right)\right)=-2$. Hence $\Sigma^{\prime}\left(D_{1}\right)$ is a twice punctured surface of genus 1 .

By inspection, $L_{1}$ is the two component link consisting of the positive trefoil and a meridian positively linking the trefoil.

Note that the link $L_{1}$ is the boundary of the surface $F$ given as the connected sum (a special case of a Murasugi sum) of the fiber surface of the positive trefoil $F_{2,3}$ and the positive Hopf band $F_{2,2}$. In particular, $F$ is a fiber surface (since Murasugi sum preserves fiberedness) for $L$ with Euler characteristic -2 . Since $F$ is a fiber surface, it is the unique Euler characteristic maximizing Seifert surface for $L_{1}$; therefore $\Sigma^{\prime}\left(D_{1}\right)$ is isotopic to $F$. However, $F$ is a quasipositive Seifert surface by (M) since it is the Murasugi sum of the two quasipositive Seifert surfaces $F_{2,3}$ and $F_{2,2}$.

- $\mathbf{6}^{\prime}\left(\mathbf{D}_{2}\right)$. This is seen to be isotopic to $\Sigma^{\prime}\left(D_{1}\right)$, for example via rotation about the vertical axis in the plane. 


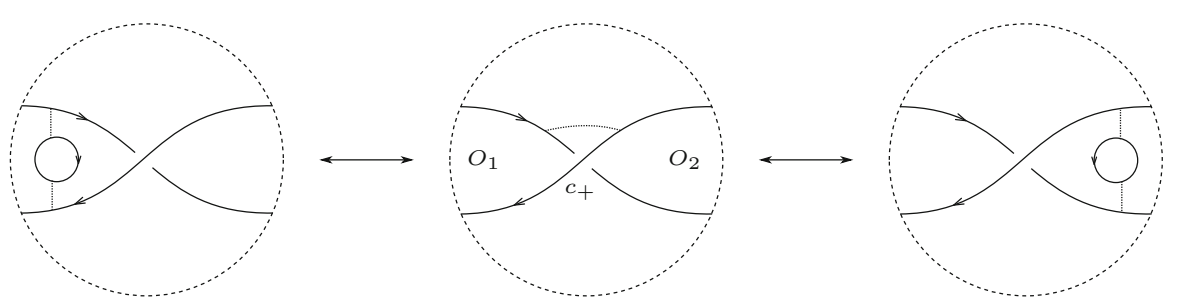

Fig. 9 Left-to-middle and right-to-middle: swapping a Seifert circle over $c_{+}$into a crossing. Left-to-right: swapping a Seifert circle from $O_{1}$ over $c_{+}$into a Seifert circle in $O_{2}$

- $\mathbf{6}^{\prime}\left(\mathbf{D}_{\mathbf{3}}\right)$. We consider a diagram move (similar to swapping a crossing) indicated in Fig. 9 that swaps a Seifert circle with two adjacent crossings into a crossing and vice versa.

Note that $\Sigma^{\prime}(D)=\Sigma^{\prime}\left(D^{\prime}\right)$ for diagrams $D$ and $D^{\prime}$ that are related by this move. We apply this move (Fig. 9(left-to-middle)) to $D_{3}$ : swap the Seifert circle in $O_{1}$ over $c_{+}$into a crossing in $U_{2}$ to get a diagram $D_{3}^{\prime}$ with two crossings in $U_{2}$ and one crossing in $U_{1}$. One of the crossings in $U_{2}$ is next to the other crossing in $U_{2}$ and also next to the crossing in $U_{1}$. By Lemma $2.7, \Sigma^{\prime}\left(D_{3}^{\prime}\right)$ is quasipositive if and only if $\Sigma^{\prime}\left(D_{3}^{\prime \prime}\right)$ is quasipositive, where $D_{3}^{\prime \prime}$ is the diagram obtained from $D_{3}^{\prime}$ by deleting the crossing in $U_{1}$ and one of the crossings in $U_{2}$.

Finally, we observe that $\Sigma^{\prime}\left(D_{3}^{\prime \prime}\right)$ is a quasipositive surface. This follows since $D_{3}^{\prime \prime}$ can be obtained from $D_{1}$ by deleting crossings and Seifert circles establishing that $\Sigma^{\prime}\left(D_{3}^{\prime \prime}\right)$ is an incompressible subsurface of the quasipositive surface $\Sigma^{\prime}\left(D_{1}\right)$.

- $\mathbf{6}^{\prime}\left(\mathbf{D}_{4}\right)$. This is seen to be isotopic to $\Sigma^{\prime}\left(D_{3}\right)$, for example via rotation about the vertical axis in the plane.

- $\mathbf{6}^{\prime}\left(\mathbf{D}_{5}\right)$. First use the move depicted in Fig. 9(middle-to-left) to swap the crossing in $U_{2}$ over $c_{+}$to result in a diagram with two Seifert circles in $O_{1}$. Then swap the Seifert circle in $O_{2}$ over $c_{+}$to $O_{1}$ using the move depicted in Fig. 9(right-to-left) resulting in a diagram $D_{5}^{\prime}$ with three Seifert circles in $O_{1}$.

The Seifert surface $\Sigma^{\prime}\left(D_{5}^{\prime}\right)$ is isotopic to $\Sigma^{\prime}\left(D_{5}\right)$ and is easily seen to be the Seifert surface of a positive diagram of the $(-2,-2,-2)$-pretzel link, and hence quasipositive.

- $\mathbf{6}^{\prime}\left(\mathbf{D}_{\mathbf{6}}\right)$. The surface $\Sigma^{\prime}\left(D_{6}\right)$ is a thrice-punctured sphere since it has Euler characteristic -1 and its boundary is a three component link $L_{6}$.

By inspection, the link $L_{6}$ is the three-component link given as an unknot with two parallel positively linked meridians. Thus, we note that $L_{6}$ is the boundary of the Seifert surface $S$ of Euler characteristic - 1 given as the connected sum of two positive Hopf bands. As for $\Sigma^{\prime}\left(D_{1}\right)$, we conclude that $S$ is a quasipositive Seifert surface that is isotopic to $\Sigma^{\prime}\left(D_{6}\right)$. Thus, $\Sigma^{\prime}\left(D_{6}\right)$ is a quasipositive Seifert surface.

- $\mathbf{6}^{\prime}\left(\mathbf{D}_{7}\right)$. The surfaces $\Sigma^{\prime}\left(D_{7}\right)$ and $\Sigma^{\prime}\left(D_{6}\right)$ are isotopic since $D_{6}$ can be turned into $D_{7}$ by swapping a crossing.

- $\mathbf{6}^{\prime}\left(\mathbf{D}_{\mathbf{8}}\right)$. The surfaces $\Sigma^{\prime}\left(D_{8}\right)$ and $\Sigma^{\prime}\left(D_{5}\right)$ are isotopic since swapping a crossing in $D_{5}$ (and then moving the crossing over infinity) turns $D_{5}$ into $D_{8}$.

We end the section with the generalization of Lemma 1.3 used above. 
(a)

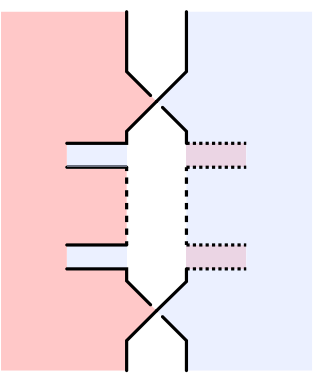

(b)

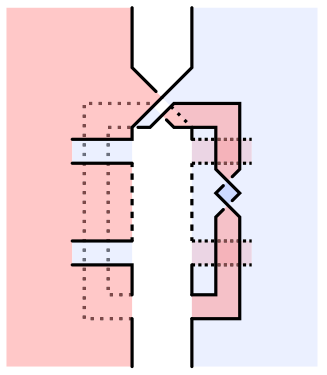

(c)

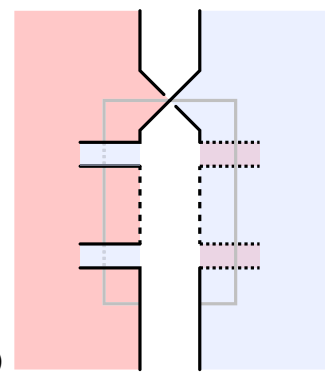

Fig. 10 Inserting a positive crossing next to another one by positive Hopf plumbing (generalizing Fig. 1). a Local picture of $\Sigma^{\prime}(D)$ containing the two ribbons corresponding to two crossings $c_{1}$ and $c_{2}$ that are next to each other. Note that the central white region could contain infinity. $\mathbf{b}$ The result of plumbing a positive Hopf band in c. c A closed interval (gray) in $\Sigma^{\prime}\left(D^{\prime}\right)$ along which a positive Hopf band gets plumbed to the blue side

Lemma 2.7 Let D be a diagram with two marked crossings of opposite sign $c_{-}$and $c_{+}$. If two positive crossings $c_{1}$ and $c_{2}$ are next to each other, then for some $i \in\{1,2\}$, the diagram $D^{\prime}$ obtained by deleting $c_{i}$ satisfies: $\Sigma^{\prime}(D)$ is a quasipositive Seifert surface if and only $\Sigma^{\prime}\left(D^{\prime}\right)$ is a quasipositive Seifert surface.

Proof of Lemma 2.7 One direction of the Lemma follows immediately since $\Sigma^{\prime}\left(D^{\prime}\right)$ is an incompressible subsurface of $\Sigma^{\prime}(D)$. The other direction shall be proven in a similar way as Lemma 1.3. We may and do assume that $c_{1}$ and $c_{2}$ are next to each other without swapping needed (otherwise swap a crossing first and consider the resulting diagram as $D$ ). Writing $k$ and $k^{\prime}$ for the generalized Seifert circles adjacent to $c_{1}$ and $c_{2}$, we wish to prove that the local situation is isotopic to Fig. 10a. Then, while there may be twisted ribbons attached to $k$ and to $k^{\prime}$ between $c_{1}$ and $c_{2}$, either all of those ribbons lie above the disk corresponding to $k$, and below the disk corresponding to $k^{\prime}$, or vice versa. So, the Hopf band in Fig. 10b may be plumbed to Fig. 10c such that it does not interfere with the ribbons.

To make this plan work, we distinguish two cases depending on whether $k$ and $k^{\prime}$ are nested or not; in each of the cases we pay attention to the possibility that $k$ and $k^{\prime}$ may be $s_{1}$ or $s_{2}$.

Case when $k$ and $k^{\prime}$ are not nested. It turns out that $D^{\prime}$ can be chosen such that $\Sigma^{\prime}(D)$ arises from plumbing a positive Hopf band to $\Sigma^{\prime}\left(D^{\prime}\right)$, which implies that $\Sigma^{\prime}(D)$ is a quasipositive Seifert surface if and only if $\Sigma^{\prime}\left(D^{\prime}\right)$ is. The argument is more involved version of the proof of Lemma 1.3; in particular, we will have to be careful which of the two crossings $c_{1}$ and $c_{2}$ to eliminate in $D$ to obtain $D^{\prime}$.

We first consider the case that at least one of the generalized Seifert circles $k$ or $k^{\prime}$ is a Seifert circle (i.e. not an $s_{i}$ ). The situation is as depicted in Fig. 10a (we note that picture only depicts a closed range of $z$-coordinate height-far above or below there could be further disks corresponding to generalized Seifert circles that contain both $k$ and $k^{\prime}$ ).

This is due to the $z$-coordinate convention of nested disks assuring that all the ribbons corresponding to crossings on $k$ and $k^{\prime}$ to the other side than the $c_{i}$ are to the positive (red) side of the disks corresponding to $k$ and $k^{\prime}$. Then, the surface can 
be isotoped to be the result (see Fig. 10b) of plumbing a positive Hopf band to the negative (blue) side of $\Sigma^{\prime}\left(D^{\prime}\right)$ (see Fig. 10c), where $D^{\prime}$ is the diagram obtained by deleting the crossing $c_{i}$ in $D$ that is depicted at the bottom of Fig. 10a.

If instead $k$ and $k^{\prime}$ are $s_{1}$ and $s_{2}$, then the crossings $c_{1}$ and $c_{2}$ lie in $U_{1}$ or $U_{2}$. If they lie in $U_{1}$, the situation is again exactly as depicted in Fig. 10a. If instead the crossings $c_{1}$ and $c_{2}$ lie in $U_{2}$, then $\Sigma^{\prime}(D)$ can be isotoped to look like Fig. 10a by locally pulling the disks corresponding to $s_{1}$ and $s_{2}$ apart, so the first is no longer above the second. Note that, different from the previous cases, the positive side of $\Sigma^{\prime}(D)$ is depicted as blue and the plumbing of the positive Hopf band happens to the positive (blue) side of $\Sigma^{\prime}\left(D^{\prime}\right)$. Again, here $D^{\prime}$ is the appropriate diagram obtained from $D$ by deleting either $c_{1}$ or $c_{2}$.

\section{Case when $k$ and $k^{\prime}$ are nested.}

First remark that $k$ or $k^{\prime}$ is a Seifert circle since $s_{1}$ and $s_{2}$ are not nested. Second, note that either the two $c_{i}$ and the two $s_{i}$ all lie to the same side of $k$, or they all lie to the same side of $k^{\prime}$. All in all, we suppose w.l.o.g. that $k$ is a Seifert circle and the $s_{i}$ and the $c_{i}$ all lie to the same side of $k$.

We now split $\Sigma^{\prime}(D)$ as a Murasugi sum along $k$. Let $D_{-}$and $D_{+}$be the link diagrams so that $D=D_{-} \cup D_{+}$and $D_{-} \cap D_{+}=k$ (as in Lemma 1.2), where we let $D_{-}$be the link diagram that contains the $s_{i}$ and the $c_{i}$. The simple case that $D_{+}$consists only of $k$ and $D_{-}=D$ is possible. The Seifert surface $\Sigma^{\prime}(D)$ is a Murasugi sum of $\Sigma\left(D_{+}\right)$ and $\Sigma^{\prime}\left(D_{-}\right)$. Since $D_{+}$has no negative crossings, $\Sigma\left(D_{+}\right)$is a quasipositive Seifert surface and, thus, $\Sigma^{\prime}(D)$ is a quasipositive Seifert surface if and only if $\Sigma^{\prime}\left(D_{-}\right)$is by (M).

We now argue that $\Sigma^{\prime}\left(D_{-}\right)$arises by positive Hopf plumbing on $\Sigma^{\prime}\left(D_{-}^{\prime}\right)$, where $D_{-}^{\prime}$ is a diagram obtained from deleting one of the $c_{i}$ in $D_{-}$. For this we note that the Seifert surface $\Sigma^{\prime}\left(D_{-}\right)$can be isotoped (by folding the disk corresponding to either $k$ or $k^{\prime}$, which ever contains the other, along the part of its boundary connecting the two ribbons corresponding to $c_{1}$ and $c_{2}$ ) to look like Fig. 10a.

In fact, the situation is necessarily simpler as depicted in Fig. 10a: on the disk corresponding to $k$ there will be no ribbons leaving between $c_{1}$ and $c_{2}$ on either side (red or blue). In other words, the situation is as depicted in Fig. 10a to one side and as depicted in Fig. 1c on the other side.

So then, as before, $\Sigma^{\prime}\left(D_{-}\right.$) is the result (see Fig. 10b) of plumbing a positive Hopf band to $\Sigma^{\prime}\left(D_{-}^{\prime}\right)$ (see Fig. 10c), where $D_{-}^{\prime}$ is the appropriate diagram obtained from $D_{-}$by deleting one of the $c_{i}$. We note that both plumbing to the positive side and plumbing to the negative side can occur.

Finally, we set $D^{\prime}$ to be the union of $D_{-}^{\prime}$ and $D_{+}$. The Seifert surface $\Sigma^{\prime}\left(D^{\prime}\right)$ is a Murasugi sum of $\Sigma\left(D_{+}\right)$and $\Sigma^{\prime}\left(D_{-}^{\prime}\right)$ and, thus, $\Sigma^{\prime}\left(D^{\prime}\right)$ is a quasipositive Seifert surface if and only if $\Sigma^{\prime}\left(D_{-}^{\prime}\right)$ is (by $(\mathrm{M})$ ). Therefore, we conclude that $\Sigma^{\prime}(D)$ is a quasipositive Seifert surface if and only if $\Sigma^{\prime}\left(D^{\prime}\right)$ is, as desired.

\section{Canonical quasipositive surfaces}

Let us start with some graph theoretic concepts. 


\section{Definition 3.1}

- A path $P$ is a sequence $e_{1}, \ldots, e_{n}$ of distinct edges in which $e_{i}$ has vertices $v_{i}^{1}$ and $v_{i}^{2}$ such that $v_{i}^{2}=v_{i+1}^{1}$ and such that every vertex appears at most twice as endpoint of an edge of $P$.

- The length of such a path $P$ is denoted by $\ell(P)=n$.

- A cycle $C$ is a path as above with $v_{n}^{2}=v_{1}^{1}$.

- A region of a plane graph $G$ is a connected component of $\mathbb{R}^{2} \backslash G$.

- A graph $G$ is 2-connected if it has at least three vertices, is connected, and the result of removing any vertex is again connected.

- A weighted graph is a graph in which each edge carries either the weight +1 or the weight -1 . For a collection $E$ of edges of a weighted graph, we denote by $w(E) \in \mathbb{Z}$ the total weight of $E$, i.e. the sum of the weights of the edges in $E$.

Our main theorem of this section is the following.

Theorem B. A canonical surface is quasipositive if and only if all cycles of its Seifert graph have strictly positive total weight.

Proof A cycle $C$ of $\Gamma(D)$ lifts to a non-null-homologous unknot in $\Sigma(D)$ with framing $w(C)$. A tubular neighborhood of that unknot in $\Sigma(D)$ is an annulus with $w(C)$ full twists, and an incompressible subsurface of $\Sigma(D)$. So if $\Sigma(D)$ is quasipositive, then $w(C)>0$ follows from (S). This establishes the necessity of the cycle condition for quasipositivity.

To see that that the cycle condition for quasipositivity is sufficient, suppose some diagram $D$ has $\Gamma(D)$ satisfying the hypothesis of Theorem B.

If $D$ is a split diagram, then $\Gamma(D)$ is not connected and the cycle condition can be checked on each connected component individually. Therefore we may and do assume that $D$ is non-split.

If there is a Seifert circle $k$ in $D$ that has non-empty interior and exterior, then $\Sigma(D)$ may be expressed as the Murasugi sum of some $\Sigma\left(D_{i}\right)$ and $\Sigma\left(D_{e}\right)$ (see Lemma 1.2). Since $\Gamma\left(D_{i}\right)$ and $\Gamma\left(D_{e}\right)$ are both subgraphs of $\Gamma(D)$, we conclude that Theorem B follows from considering diagrams where each Seifert circle either has empty interior or empty exterior.

By moving infinity we move to a different diagram but with an isotopic canonical surface. So, by possibly moving infinity, we may and do assume that every Seifert circle of $D$ has empty interior.

Hence we have reduced the proof to Proposition 3.2.

Proposition 3.2 If $D$ is a non-split link diagram such that every Seifert circle of $D$ has empty interior, and such that all cycles of $\Gamma(D)$ have positive total weight, then $\Sigma(D)$ is quasipositive.

Recall from the paragraph after Theorem B in Sect. 1 that if $D$ is a link diagram with all Seifert circles having empty interior, then its Seifert graph $\Gamma(D)$ is naturally a plane graph, while also being bipartite and weighted. All graphs considered in this section will be bipartite weighted plane graphs. 
Proof of Proposition 3.2 Let $D$ be a link diagram satisfying the hypothesis of the proposition. Since $D$ is non-split, $\Gamma(D)$ is connected.

Suppose that $D$ is a connected sum of diagrams $D_{1}$ and $D_{2}$, each with at least one crossing. Then $\Sigma(D)$ is a connected sum (a special case of a Murasugi sum) of $\Sigma\left(D_{1}\right)$ and $\Sigma\left(D_{2}\right)$ and it suffices to consider the summands by $(\mathrm{M})$. Therefore we only consider diagrams that are not such connected sums.

If $D$ has only one Seifert circle, $\Sigma(D)$ is a disk, which is quasipositive. Similarly if $D$ has only two Seifert circles and only one crossing.

If $D$ has two Seifert circles and $n \geq 2$ crossings, then each crossing is positive since otherwise the cycle positivity condition of $\Gamma(D)$ would be violated. Then we see that $\Sigma(D)$ is the fiber surface of the positive $(2, n)$-torus link, which is quasipositive. This case will be the root case of a proof by induction.

We assume now that $D$ has at least three Seifert circles. Since $D$ is not a non-trivial connected sum, $\Gamma(D)$ is $2-$ connected (see Definition 3.1). Then it is a straightforward graph theoretic result about $2-$ connected plane graphs that the boundary of each region of $\mathbb{R}^{2} \backslash \Gamma(D)$ is a cycle. We will call such a cycle boundary cycle.

Our strategy is a proof by induction over the following measure of complexity of $\Gamma(D)$. Suppose $x=\left(x_{1}, x_{2}, x_{3}, \ldots\right)$ and $y=\left(y_{1}, y_{2}, y_{3}, \ldots\right)$ are two infinite sequences of integers with only finitely many non-zero integers. Define $x>y$ iff the rightmost non-zero entry of $x-y$ is positive. Let $f_{i}$ be the number of boundary cycles of length $2 i$. We define the infinite sequence

$$
f(\Gamma(D))=\left(f_{1}, f_{2}, f_{3}, \ldots\right)
$$

Given a link diagram $D$ satisfying the hypothesis of Proposition 3.2, our idea is to produce a new link diagram $D^{\prime}$ also satisfying the hypothesis. Furthermore we aim to do this so that $f\left(\Gamma\left(D^{\prime}\right)\right)<f(\Gamma(D))$ and so that $\Sigma(D)$ is a quasipositive surface if $\Sigma\left(D^{\prime}\right)$ is a quasipositive surface. Having already verified the root case that all boundary cycles have length 2 (which implies having two Seifert circles), the induction will give us the result.

If $\Gamma(D)$ contains a vertex of degree 2 adjacent to a positive and a negative edge, then we have $\Sigma(D)=\Sigma\left(D^{\prime}\right)$ where $D^{\prime}$ is obtained from $D$ by removing two crossings via a Reidemeister II move. Furthermore $D^{\prime}$ satisfies the hypothesis of Proposition 3.2 and has $f\left(\Gamma\left(D^{\prime}\right)\right)<f(\Gamma(D))$. So we may and do assume that $\Gamma(D)$ contains no degree 2 vertices adjacent to both a positive and negative edge.

Let us now introduce a new move, which generalizes (7) from the proof of Theorem A. Suppose $v, w$ are vertices of $\Gamma(D)$ on the boundary $C$ of a region of $\mathbb{R}^{2} \backslash \Gamma(D)$. Let $d$ be the distance (lengthwise, not weighted) between $v$ and $w$ along $C$ and suppose $d \geq 2$. We now describe a diagram $D^{\prime}$ obtained from $D$. It is enough to describe $\Gamma\left(D^{\prime}\right)$, which is obtained from $\Gamma(D)$ by adding a chord consisting of a path of $(d-2)$ positive edges between $v$ and $w$ inside of the region. In the special case of $d=2$, adding a chord of length 0 is understood as merging $v$ and $w$. The two crucial observations are:

- We have that $f\left(\Gamma\left(D^{\prime}\right)\right)<f(\Gamma(D))$ because the regions of $\mathbb{R}^{2} \backslash \Gamma\left(D^{\prime}\right)$ correspond to those of $\mathbb{R}^{2} \backslash \Gamma(D)$, except for the region with boundary $C$, which is split into two regions, each of them with strictly fewer edges than $C$. 
- The surface $\Sigma(D)$ is an incompressible subsurface of $\Sigma\left(D^{\prime}\right)$.

So to conclude the proof of the proposition, it suffices to show that this move is always possible in such a way that $D^{\prime}$ still satisfies the hypothesis of the proposition. Note that the only cycles of $\Gamma\left(D^{\prime}\right)$ not occurring as cycles of $\Gamma(D)$ are those that pass through the new chord. So it suffices to pick $v, w$ and $C$ such that any path $Q$ in $\Gamma(D)$ between $v$ and $w$ satisfies $w(Q)+d-2 \geq 2$. That this is always possible is the contents of Propositions 3.5 and 3.6.

Definition 3.3 We say that a link diagram $D$ has property (*) if it satisfies the following.

- All Seifert circles of $D$ have empty interior.

- All cycles of $\Gamma(D)$ have positive total weight.

- $\Gamma(D)$ is 2-connected (see Definition 3.1).

- $\Gamma(D)$ contains no degree 2 vertices adjacent to both a positive and a negative edge.

Definition 3.4 Suppose that $D$ has property (*) and $C$ is the boundary cycle of a region of $\mathbb{R}^{2} \backslash \Gamma(D)$. We say that $C$ is splittable if there exist vertices $v$ and $w$ of $C$, distance $d \geq 2$ apart on $C$, such that every path $Q$ in $\Gamma(D)$ connecting $v$ to $w$ satisfies $w(Q) \geq 4-d$.

Proposition 3.5 Suppose that D has property (*). Then there is a region of $\mathbb{R}^{2} \backslash \Gamma(D)$ whose boundary cycle $C$ has $w(C) \geq 4$.

We postpone the proof of this proposition to the end of the section.

Proposition 3.6 Suppose that D has property (*) and $C$ is the boundary cycle of a region of $\mathbb{R}^{2} \backslash \Gamma(D)$ with $w(C) \geq 4$. Then $C$ is splittable.

Proof The proof is divided into two cases given as Lemmas 3.7 and 3.8.

Lemma 3.7 Suppose that $D$ has property (*), and that $C$ is the boundary cycle of a region of $\mathbb{R}^{2} \backslash \Gamma(D)$ with $w(C) \geq 4$ and at least one edge of $C$ negative. Then $C$ is splittable.

Lemma 3.8 Suppose that $D$ has property (*), and that $C$ is the boundary cycle of a region of $\mathbb{R}^{2} \backslash \Gamma(D)$ with $w(C) \geq 4$ and every edge of $C$ positive. Then $C$ is splittable.

For the proof of Lemmas 3.7 and 3.8 we first collect some straightforward facts, without proof, in the following lemma.

Lemma 3.9 We have the following.

(1) Suppose that $H$ is a graph and $v$ and $w$ are distinct vertices of $H$. Then paths from $v$ to $w$ are exactly the minimal subgraphs of $H$ in which $v$ and $w$ are the only vertices with odd degree.

(2) If $H$ is a graph with vertices of only even degree then its set of edges can be written as a disjoint union of cycles. 
(3) Suppose that $H$ is a graph with exactly two vertices $v$ and $w$ of odd degree and that $P$ is any path from $v$ to $w$ (such a $P$ exists by the first part of this lemma). Then removing the set of edges of $P$ from $H$ leaves a graph whose set of edges is a disjoint union of cycles.

Proof of Lemma 3.7 Let us pick $v$ and $w$ as follows. Walking around $C$, pick $v$ such that the next edge is positive and the one immediately after is negative, and continue walking until the next positive edge, and call its farther vertex $w$. Then one has walked along a path $P$ with $w(P)=4-\ell(P)$. The other path $P^{\prime}$ in $C$ between $v$ and $w$ satisfies $w\left(P^{\prime}\right)+w(P) \geq 4$, and so $\ell\left(P^{\prime}\right) \geq w\left(P^{\prime}\right) \geq 4-w(P)=\ell(P)$. Hence $P$ is not the longer path between $v$ and $w$ around $C$, and $d=\ell(P)$.

For every path $Q$ in $\Gamma(D)$ between $v$ and $w$, we must show that $w(Q) \geq 4-d$, which is equivalent to showing $w(Q) \geq w(P)$ since $4-d=4-\ell(P)=w(P)$.

So let $Q$ be such a path. Note that $(P \cup Q) \backslash(P \cap Q)$ is a union of cycles (see Lemma 3.9(2)), say $Z_{1}, \ldots, Z_{n}$. Let us write $Z_{i}=P_{i} \sqcup Q_{i}$ for a decomposition of each $Z_{i}$ into sets of edges $P_{i} \subset P$ and $Q_{i} \subset Q$. Then we have that

$$
\begin{aligned}
& w(P)=w\left(P_{1}\right)+\cdots+w\left(P_{n}\right)+w(P \cap Q), \\
& w(Q)=w\left(Q_{1}\right)+\cdots+w\left(Q_{n}\right)+w(P \cap Q) .
\end{aligned}
$$

So we shall be done if we can show that $w\left(Q_{i}\right) \geq w\left(P_{i}\right)$ for all $i$.

Since $P$ contains only two positive edges, we must have $w\left(P_{i}\right) \leq 2$, with equality only when $P_{i}$ consists of exactly the two positive edges of $P$ (in other words the first and last edge of $P$ ). Since the path $Q_{i}$ of course cannot enter the region bounded by $C$ the only way that $w\left(P_{i}\right)=2$ can happen is if $Q_{i}$ consists of a path connecting $v$ to $w$ and another path connecting the other two endpoints of the first and last edge of $P$. But $Q$ is a path connecting $v$ to $w$ and $Q_{i} \subset Q$, so we have a contradiction by Lemma 3.9(1). Therefore we must have $w\left(P_{i}\right) \leq 1$ for all $i$. Further note that $w\left(Q_{i}\right)+w\left(P_{i}\right)=w\left(Z_{i}\right) \geq 2$ since $Z_{i}$ is a cycle. Hence we can conclude that $w\left(Q_{i}\right) \geq w\left(P_{i}\right)$ for all $i$.

A heuristic important for our proof of Lemma 3.8 is that if two paths of a weighted graph intersect, one can resolve them and obtain two new paths which have the same total weight. This heuristic turns out, when formalized in the following lemma, only to yield an inequality rather than an equality.

Lemma 3.10 Let $D$ be a diagram which has property (*) and consider the boundary cycle $C$ of a region of $\mathbb{R}^{2} \backslash \Gamma(D)$. Suppose that $\ell(C) \geq 4$, and let $v_{i}$ be distinct vertices of $C$ for $i \in \mathbb{Z} / 4$, occurring in the cyclic ordering around $C$. Suppose that $P_{02}$ and $P_{13}$ are paths from $v_{0}$ to $v_{2}$ and from $v_{1}$ to $v_{3}$ respectively.

Then for some $i \in\{0,1\}$ there exists a path $P_{i, i+1}$ from $v_{i}$ to $v_{i+1}$ and a path $P_{i+2, i+3}$ from $v_{i+2}$ to $v_{i+3}$ such that

$$
w\left(P_{i, i+1}\right)+w\left(P_{i+2, i+3}\right) \leq w\left(P_{02}\right)+w\left(P_{13}\right) .
$$


Proof Consider the subgraph $H$ of $\Gamma(D)$

$$
H=\left(P_{02} \cup P_{13}\right) \backslash\left(P_{02} \cap P_{13}\right) .
$$

The vertices in $H$ of odd degree are exactly the vertices $v_{i}$ for $i \in \mathbb{Z} / 4$. Let $\widetilde{H}$ be the subgraph of $H$ obtained by removing those connected components of $H$ not containing any of the vertices $v_{i}$. Again the vertices of $\widetilde{H}$ of odd degree are exactly the vertices $v_{i}$. Therefore $\widetilde{H}$ is either connected or has exactly two components each containing two of the vertices $P_{i}$. Any path connecting $v_{0}$ and $v_{2}$ must intersect any path connecting $v_{1}$ and $v_{3}$. Hence, it cannot be the case that there are two components of which one contains $v_{0}$ and $v_{2}$ and while the other contains $v_{1}$ and $v_{3}$. Therefore $v_{0}$ is in the same component as $v_{1}$ or as $v_{3}$. Let us assume the former for now.

Let $Q$ be a path in $\widetilde{H}$ connecting $v_{0}$ to $v_{1}$. Note that $Q$ is a subgraph of $H$ and so, by construction, each edge of $Q$ occurs either in $P_{02}$ or in $P_{13}$ but not in both. Therefore we have

$$
w\left(P_{02}\right)+w\left(P_{13}\right)=w\left(\left(P_{02} \cup Q\right) \backslash\left(P_{02} \cap Q\right)\right)+w\left(\left(P_{13} \cup Q\right) \backslash\left(P_{13} \cap Q\right)\right) .
$$

Now $v_{1}$ and $v_{2}$ are exactly the vertices of $\left(P_{02} \cup Q\right) \backslash\left(P_{02} \cap Q\right)$ of odd degree. Therefore $\left(P_{02} \cup Q\right) \backslash\left(P_{02} \cap Q\right)$ can be written as the disjoint union of a path $P_{12}$ from $v_{1}$ to $v_{2}$ and some cycles (by Lemma 3.9). Since by assumption each cycle has weight $\geq 2$, we must have that

$$
w\left(P_{12}\right) \leq w\left(\left(P_{02} \cup Q\right) \backslash\left(P_{02} \cap Q\right)\right) .
$$

Similarly there is a path $P_{30}$ from $v_{3}$ to $v_{0}$ satisfying

$$
w\left(P_{30}\right) \leq w\left(\left(P_{13} \cup Q\right) \backslash\left(P_{13} \cap Q\right)\right) .
$$

Hence we have

$$
\begin{aligned}
w\left(P_{12}\right)+w\left(P_{30}\right) & \leq w\left(\left(P_{02} \cup Q\right) \backslash\left(P_{02} \cap Q\right)\right)+w\left(\left(P_{13} \cup Q\right) \backslash\left(P_{13} \cap Q\right)\right) \\
& =w\left(P_{02}\right)+w\left(P_{13}\right) .
\end{aligned}
$$

The other case follows from making the assumption that $v_{0}$ is in the same component of $\widetilde{H}$ as $v_{3}$.

Lemma 3.11 Suppose that D has property (*) and that $C$ is the boundary of a region of $\mathbb{R}^{2} \backslash \Gamma(D)$ such that all edges of $C$ have weight +1 . Let $v$ and $w$ be vertices of $C$ such that the shortest path in $C$ between $v$ and $w$ has length $d \geq 1$. Then there is no path between $v$ and $w$ of weight less than $2-d$.

Proof Let $P$ be a path between $v$ and $w$. Let us write $Q$ for a path of length $d$ contained in $C$ connecting $v$ to $w$. Consider $P \cap Q$. Each connected component of $P \cap Q$, since it is a subgraph of $Q$, is a path in $C$. Furthermore the set of edges of $(P \cup Q) \backslash(P \cap Q)$ 
can be written as a disjoint union of cycles. Let us write $c$ for the number of cycles in such a decomposition of $(P \cup Q) \backslash(P \cap Q)$.

In the case that $c=0$ then $P=Q$ and $w(Q)=w(P)=d \geq 2-d$.

In the case that $c \geq 1$ then

$$
\begin{aligned}
w(P) & =w((P \cup Q) \backslash(P \cap Q))+2 w(P \cap Q)-w(Q) \\
& \geq 2 c+2 w(P \cap Q)-d \geq 2 c-d \geq 2-d .
\end{aligned}
$$

Now we are in a position to give the proof of Lemma 3.8, thus establishing Proposition 3.6.

Proof of Lemma 3.8 Let us proceed by contradiction. So assume that $C$ is a boundary cycle with no negative edges, of total weight $w(C)=\ell(C)=2 n \geq 4$, and assume $C$ is not splittable. That is to say that for any pair of vertices $v, w$ on $C$ of distance $d$ along $C$, there is a path $Q$ in $\Gamma(D)$ with $w(Q)<4-d$, thus $2 n \leq 2-d$.

Pick $v_{i}$ for $i \in \mathbb{Z} / 4$ on $C$ in the cyclic ordering, such that the distances between $v_{i}$ and $v_{i+1}$ are $1,1, n-1$ and $n-1$ for $i=0,1,2,3$. Thus there are paths $P_{02}$ and $P_{13}$ from $v_{0}$ to $v_{2}$ and from $v_{1}$ to $v_{3}$, respectively, such that $w\left(P_{02}\right) \leq 0$ and $w\left(P_{13}\right) \leq 2-n$. By Lemma 3.11 it follows that in fact $w\left(P_{02}\right)=0$ and $w\left(P_{13}\right)=2-n$. By Lemma 3.10, there are paths $P_{i, i+1}$ and $P_{i+2, i+3}$ for some $i \in\{0,1\}$ such that $w\left(P_{i, i+1}\right)+w\left(P_{i+2, i+3}\right) \leq 2-n$. This contradicts the fact that by positivity of cycle weights, $w\left(P_{i, i+1}\right)>-1$ and $w\left(P_{i+2, i+3}\right)>1-d$ and thus $w\left(P_{i, i+1}\right) \geq 1$ and $w\left(P_{i+2, i+3}\right) \geq 3-d$.

Finally, we turn to Proposition 3.5, to whose proof we devote the remainder of this section.

Proof of Proposition 3.5 We divide the proof of this proposition into Lemmas 3.14 and 3.15 .

Let us first prove the following.

Lemma 3.12 Suppose that D is a diagram which has property (*). Suppose further that the boundaries of all regions of $\mathbb{R}^{2} \backslash \Gamma(D)$ have total weight 2 . Then there is a positive vertex of $\Gamma(D)$, in other words a vertex not adjacent to a negative edge.

Proof As before, let $f_{i}$ be the number of boundary cycles of length $2 i$. Let $f$ be the total number of regions, $e$ the number of edges, $e_{-}$the number of negative edges and $v$ the number of vertices. Then we have

$$
f=\sum_{i=2}^{\infty} f_{2 i} \text { and } e=\sum_{i=2}^{\infty} i f_{2 i} \quad \text { so that } \quad v=2+\sum_{i=2}^{\infty}(i-1) f_{2 i}
$$

Then, since every region of $\mathbb{R}^{2} \backslash \Gamma(D)$ has two more positive edges in its boundary than negative edges, we have 


$$
e_{-}=\sum_{i=2}^{\infty}(i-1) f_{2 i} / 2 \text { and so } v>2 e_{-} .
$$

Definition 3.13 Let $D$ be a diagram which has property $(*)$ and let $v$ be a positive vertex of $\Gamma(D)$. Let $C_{1}, \ldots, C_{n}$ be the boundaries of those regions of $\mathbb{R}^{2} \backslash \Gamma(D)$ adjacent to $v$. We say that $v$ is a wicked positive vertex if $C_{i} \cap C_{j}$ is connected for all $i, j$.

Lemma 3.14 Suppose that D is a diagram which has property (*) and which contains a wicked positive vertex. Then $\mathbb{R}^{2} \backslash \Gamma(D)$ contains a region whose boundary cycle has weight at least 4.

Proof For a contradiction, suppose that $D$ is a diagram which has property $(*)$ and in which there is no region of $\mathbb{R}^{2} \backslash \Gamma(D)$ whose boundary has weight 4 or greater, and suppose that $v$ is a wicked positive vertex of $\Gamma(D)$.

Let $C_{1}, \ldots, C_{n}$ be the boundaries of those regions of $\mathbb{R}^{2} \backslash \Gamma(D)$ adjacent to $v$, in counterclockwise order around $v$, where the subscripts are considered modulo $n$. If all of the $C_{i}$ had length 2 , then $v$ would have $n$ edges adjacent to a vertex $w$, and no further edges, which would contradict property (*). Since $C_{i} \cap C_{i+1}$ is connected, it is a path starting at $v$ (or containing $v$ in case $n=2$ ). Since $D$ has property (*), all these paths contain only positive edges, and thus have positive total weight. Now, using that not all $C_{i}$ have length 2 , the edges of

$$
\left(C_{1} \cup \cdots \cup C_{n}\right) \backslash \bigcup_{i}\left(C_{i} \cap C_{i+1}\right)
$$

form a cycle $C$ with

$$
\begin{aligned}
w(C) & =\sum_{i} w\left(C_{i}\right)-2 \sum_{i} w\left(C_{i} \cap C_{i+1}\right) \\
& =2 n-2 \sum_{i} w\left(C_{i} \cap C_{i+1}\right) \\
& \leq 2 n-2 n=0 .
\end{aligned}
$$

But this contradicts the property (*).

Lemma 3.15 If $D$ is diagram which has property (*) then either $\Gamma(D)$ has a wicked positive vertex, or $\mathbb{R}^{2} \backslash \Gamma(D)$ contains a region whose boundary cycle has weight at least 4.

Proof of Lemma 3.15 Suppose for a contradiction that there exists at least one diagram with property $(*)$ whose Seifert graph contains no positive wicked vertices and no boundaries of weight at least 4 . Consider such diagrams with the minimal number of positive vertices, and let $D$ be one of these with the minimal number of crossings. 


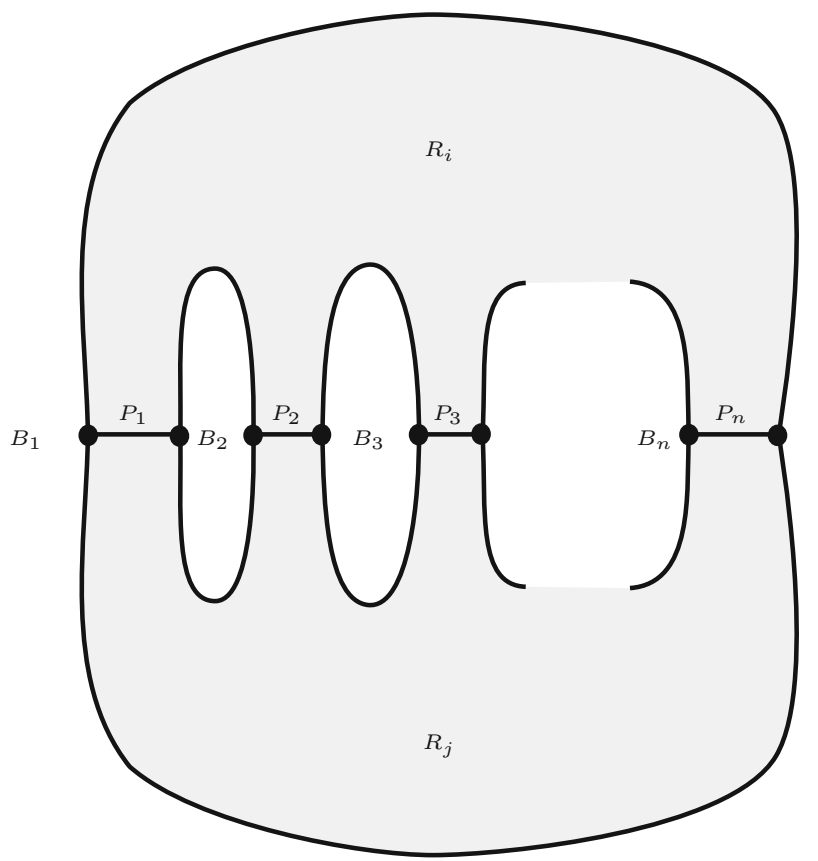

Fig. 11 A diagram of the situation of Lemma 3.15

We know by Lemma 3.12 that $D$ has a positive vertex; let us call it $v$. Let $C_{1}, \ldots, C_{n}$ be the boundaries of those regions of $\mathbb{R}^{2} \backslash \Gamma(D)$ adjacent to $v$, in counterclockwise order around $v$, where the subscripts are considered modulo $n$. Then, since $v$ is not wicked by assumption, for some $i \neq j$ we have that $C_{i} \cap C_{j}$ has $m \geq 2$ components (note that one of these components contains the positive vertex $v$ ). We write $R_{i}$ and $R_{j}$ for the closed bounded regions of $\mathbb{R}^{2}$ whose boundaries are $C_{i}$ and $C_{j}$ respectively. Then $\mathbb{R}^{2} \backslash\left(R_{i} \cup R_{j}\right)$ has $m$ components $B_{1}, \ldots, B_{n}$ and the boundary of each $\overline{B_{k}}$ is a cycle in $\Gamma(D)$. Let us write $Z_{k}$ for the boundary of $\overline{B_{k}}$, and $P_{1}, P_{2}, \ldots, P_{n}$ for the paths which are the components of $R_{i} \cap R_{j}$.

The situation is illustrated in Fig. 11. Note that the interiors of the regions $R_{i}$ and $R_{j}$ do not contain any vertices or edges of $\Gamma(D)$. Note also that some paths $P_{k}$ could consist of single vertices. Note further that the vertices of each $P_{k}$ which are not endpoints of $P_{k}$ have degree 2 in $\Gamma(D)$. Since $\Gamma(D)$ has property $(*)$ it follows that no $P_{k}$ has both positive and negative edges.

Now for any $k$ consider the subgraph of $\Gamma(D)$ that lies within $\overline{B_{k}}$. This is the Seifert graph of a diagram $D^{\prime}$ that also satisfies property $(*)$, apart from possibly containing a degree 2 vertex adjacent to both a positive and a negative edge. By Reidemeister II moves $D^{\prime}$ may be converted to a diagram $D^{\prime \prime}$ with property (*) such that $D^{\prime \prime}$ has the same number of Seifert circles as $D^{\prime}$ and possibly fewer crossings. Hence by induction $\mathbb{R}^{2} \backslash \Gamma\left(D^{\prime \prime}\right)$ contains a region whose boundary has weight 4 or more. However the regions of $\mathbb{R}^{2} \backslash \Gamma\left(D^{\prime \prime}\right)$ are in an obvious correspondence with those of $\mathbb{R}^{2} \backslash \Gamma\left(D^{\prime}\right)$ under which the weights the boundary cycles are invariant. Hence $\mathbb{R}^{2} \backslash \Gamma\left(D^{\prime}\right)$ contains 
a region whose boundary has weight 4 or more. We conclude that either $\mathbb{R}^{2} \backslash \Gamma(D)$ does as well (and we are done) or that $w\left(Z_{k}\right) \geq 4$.

We assume for a contradiction that we have $w\left(C_{k}\right)=2$ for all $C_{k}$. Then we have

$$
\begin{aligned}
2\left(w\left(P_{1}\right)+\cdots+w\left(P_{n}\right)\right) & =2 w\left(C_{i} \cap C_{j}\right) \\
& =w\left(C_{i}\right)+w\left(C_{j}\right)-\left(w\left(Z_{1}\right)+\cdots+w\left(Z_{n}\right)\right) \\
& =4-\left(w\left(Z_{1}\right)+\cdots+w\left(Z_{n}\right)\right) \\
& \leq 4-4 n \leq-2 n
\end{aligned}
$$

where the last inequality is because $n \geq 2$.

Since no $P_{k}$ has both positive and negative edges, and at least one of the $P_{k}$, say $P_{\alpha}$, (that containing the vertex $v$ ) contains no positive edge, it follows that at least one of the $P_{i}$ contains two consecutive negative edges. Let $w$ be the midpoint of these two consecutive edges. Note that there is an embedded circle in $\mathbb{R}^{2}$ which meets $\Gamma(D)$ only at $v$ and $w$. Flyping along this circle to move one of the negative edges to lie adjacent to $v$ creates a new graph which is the Seifert graph $\Gamma\left(D^{\prime}\right)$ of a diagram $D^{\prime}$.

Now, in the case that the path $P_{\alpha}$ contains at least one edge, $D^{\prime}$ admits simplification via a Reidemeister II move to a new diagram $D^{\prime \prime}$ such that $D^{\prime \prime}$ has property (*). Furthermore $\Gamma\left(D^{\prime \prime}\right)$ has either the same number or one fewer positive vertices than $\Gamma(D)$. Hence by assumption, $\Gamma\left(D^{\prime \prime}\right)$ must contain a wicked positive vertex and so by Lemma 3.14 contains a region whose boundary cycle has weight 4 .

But there is an obvious correspondence between the regions of $\mathbb{R}^{2} \backslash \Gamma\left(D^{\prime \prime}\right)$ and those of $\mathbb{R}^{2} \backslash \Gamma(D)$, and the weight of the boundary cycle of each region is preserved under this correspondence. Hence we get a contradiction in this case.

Finally consider the remaining case that the path $P_{\alpha}$ contains no edges. In this case we have that $\Gamma\left(D^{\prime}\right)$ has property $(*)$, has no wicked positive vertices, and has one fewer positive vertices than does $\Gamma(D)$. Hence by assumption $\mathbb{R}^{2} \backslash \Gamma\left(D^{\prime}\right)$ must contain a region whose boundary cycle has weight at least 4 . But again, there is a weight-preserving correspondence between the regions of $\mathbb{R}^{2} \backslash \Gamma\left(D^{\prime}\right)$ and those of $\mathbb{R}^{2} \backslash \Gamma(D)$. Hence we have a contradiction.

\section{Applications}

This section contains the proofs of the applications of Theorem B mentioned in the introduction.

Corollary C. Let $\Sigma$ be a Seifert surface that is isotopic to a canonical surface. Then $\Sigma$ is quasipositive if and only if every unknot contained in $\Sigma$ bounds a disk in $\Sigma$ or has negative induced framing by $\Sigma$.

Proof For the 'only if' direction (for which the assumption that $\Sigma$ is isotopic to a canonical surface is not necessary), assume $\Sigma$ is quasipositive and let $\gamma \subset \Sigma$ be an unknot that does not bound a disk in $\Sigma$, with framing $k$ induced by $\Sigma$. Let $B$ be an annular neighborhood of $\gamma$ in $\Sigma$. As an incompressible subsurface of $\Sigma, B$ is itself quasipositive. But as an unknotted band, $B$ is quasipositive only if its core curve $\gamma$ has 
negative induced framing by $B$ (which equals the framing induced by $\Sigma$ ). It follows that $k<0$, concluding the proof of the 'only if' direction.

For the 'if' direction, let $\Sigma$ be a canonical Seifert surface. By Theorem B, it suffices to check that all cycles in the Seifert graph of $\Sigma$ have strictly positive total weight. For a cycle $c$ in the Seifert graph, we let $A_{c}$ be the embedded annulus in $\Sigma$ given by the union of Seifert circles and half-bands that correspond to the vertices and edges that make up $c$. Note that $A_{c}$ is an unknotted annulus and incompressible in $\Sigma$. So, by assumption, $A_{c}$ has strictly negative framing. Twice the framing of $A_{c}$ equals minus the total weight of $c$. To see this, note that the framing is calculated as the linking of the two boundary components of $A_{c}$ where the orientation is reversed on one component, hence every positive crossing traversed by $A_{c}$ contributes $-\frac{1}{2}$ to the framing, while it contributes +1 to the total weight of $c$ (and analogously for negative crossings). With this we established that all cycles have strictly positive total weight, as required.

Recall that by $y$ we denote a slice-torus invariant, as defined in the introduction.

Theorem D. If $K$ is a knot with a canonical surface $\Sigma$ such that $y(K)=\operatorname{genus}(\Sigma)$, then $\Sigma$ is a quasipositive Seifert surface; in particular, $K$ is a strongly quasipositive knot.

Proof Let $g$ denote the genus of $\Sigma$. Assume toward a contradiction that $\Sigma$ is not strongly quasipositive. Then, by Corollary C, $\Sigma$ contains a homologically non-trivial unknot $U$ whose framing induced by $\Sigma$ is some non-negative integer $k$. Choose a closed disk $D \subset S^{3}$ such that $D$ intersects $\Sigma$ transversely in a proper arc $I \subset \Sigma$ that lies in the interior of $D$ and, in $\Sigma, I$ intersects $U$ transversely in exactly one point. Let $\Sigma^{\prime} \subset S^{3}$ be the surface obtained from $\Sigma$ by a $\pm 1 / k$ surgery along $\partial D$, where the sign is chosen such that $U \subset \Sigma^{\prime}$ has framing 0 induced by $\Sigma^{\prime}$. Note that one gets from the knot $K=\partial \Sigma$ to the knot $J:=\partial \Sigma^{\prime}$ by $k$ crossing changes from negative to positive, and so $y(K) \leq y(J)$. Hence ambient surgery in $B^{4}$ of $\Sigma^{\prime}$ along $U$ (i.e. replacing an annular neighborhood of $U$ in $\Sigma^{\prime}$ by two discs properly embedded in $B^{4}$ ) produces a slice surface $F$ of $J$ with genus $(F)=\operatorname{genus}\left(\Sigma^{\prime}\right)-1=g-1$. It follows that $y(K) \leq y(J) \leq g-1$, contradicting the assumption $y(K)=g$.

Corollary E. Let $K$ be a knot with $\tilde{g}(K)=g(K)$, i.e. a knot for which the genus $g(K)$ $i$ realized by a canonical surface $\Sigma$. The following are equivalent:

(1) $\Sigma$ is quasipositive,

(2) $K$ is strongly quasipositive,

(3) for $K$ the Bennequin-inequality is an equality, and

(4) $y(K)=g(K)$.

Proof $(1) \Rightarrow(2)$ holds by definition; (2) $\Rightarrow(3)$ is implied by writhe $(\beta)-n+1=2 g(K)$ for a strongly quasipositive braid $\beta$ on $n$ strands whose closure is $K$; (3) $\Rightarrow$ (4) follows since $\frac{\mathrm{sl}(K)+1}{2} \leq y(K) \leq g(K)$ holds for all knots $K$; and $(4) \Rightarrow(1)$ is immediate from Theorem D.

Acknowledgements The authors thank Sebastian Baader both generally for his advocacy of quasipositivity and specifically for a conversation that inspired an important step in the proof of Theorem B. They also thank an anonymous referee for a suggestion which lead to further applications of Theorem B. Peter Feller 
gratefully acknowledges support by the SNSF Grant 181199. Lukas Lewark is supported by the DFG, project no. 412851057.

Funding Open access funding provided by Swiss Federal Institute of Technology Zurich

\section{Declarations}

Conflict of interest On behalf of all authors, the corresponding author states that there is no conflict of interest and there is no further data.

Open Access This article is licensed under a Creative Commons Attribution 4.0 International License, which permits use, sharing, adaptation, distribution and reproduction in any medium or format, as long as you give appropriate credit to the original author(s) and the source, provide a link to the Creative Commons licence, and indicate if changes were made. The images or other third party material in this article are included in the article's Creative Commons licence, unless indicated otherwise in a credit line to the material. If material is not included in the article's Creative Commons licence and your intended use is not permitted by statutory regulation or exceeds the permitted use, you will need to obtain permission directly from the copyright holder. To view a copy of this licence, visit http://creativecommons.org/licenses/by/4.0/.

\section{References}

1. Baader, S.: Quasipositivity and homogeneity. Math. Proc. Camb. Philos. Soc. 139(2), 287-290 (2005)

2. Baader, S.: Slice and Gordian numbers of track knots. Osaka J. Math. 42, 257-271 (2005). arXiv:math/0504594

3. Baader, S., Ishikawa, M.: Legendrian graphs and quasipositive diagrams. Ann. Fac. Sci. Toulouse Math. (6) 18(2), 285-305 (2009). arXiv:math/0609592

4. Baader, S., Ishikawa, M.: Legendrian framings for two-bridge links. Proc. Am. Math. Soc. 139(12), 4513-4520 (2011). arXiv:0910.0355

5. Bennequin, D.: Entrelacements et équations de Pfaff. Astérisque 107-108, 87-161 (1983)

6. Boileau, M., Orevkov, S.: Quasi-positivité d'une courbe analytique dans une boule pseudo-convexe. C. R. Acad. Sci. Paris Sér. I Math. 332(9), 825-830 (2001)

7. Cromwell, P.R.: Homogeneous links. J. Lond. Math. Soc. 39(3), 535-552 (1989)

8. Crowell, R.: Genus of alternating link types. Ann. Math. 69, 258-275 (1959)

9. Gabai, D.: Genera of the arborescent links. Mem. Am. Math. Soc. 59(339), i-viii and 1-98 (1986)

10. Hamer, J., Ito, T., Kawamuro, K.: Positivities of knots and links and the defect of Bennequin inequality, Exp. Math. Published online, 1-27 (2019). arXiv:1809.10836

11. Hedden, M., Ording, P.: The Ozsváth-Szabó and Rasmussen concordance invariants are not equal. Am. J. Math. 130(2), 441-453 (2008). arXiv:math/0512348

12. Hedden, M.: Notions of positivity and the Ozsváth-Szabó concordance invariant. J. Knot Theory Ramif. 19(5), 617-629 (2010). arXiv:math/0509499

13. Kronheimer, P.B., Mrowka, T.S.: Gauge theory for embedded surfaces, I. Topology 32(4), 773-826 (1993)

14. Lewark, L.: Rasmussen's spectral sequences and the $\mathfrak{s l}_{n}$-concordance invariants. Adv. Math. 260, 59-83 (2014). arXiv:1310.3100

15. Livingston, C.: Computations of the Ozsváth-Szabó knot concordance invariant. Geom. Topol. 8, 735-742 (2004). arXiv:math/0311036

16. Morton, H.R.: Seifert circles and knot polynomials. Math. Proc. Camb. Philos. Soc. 99(1), 107-109 (1986)

17. Murasugi, K.: On the genus of the alternating knot. I, II. J. Math. Soc. Jpn. 10(94-105), 235-248 (1958)

18. Nakamura, T.: Four-genus and unknotting number of positive knots and links. Osaka J. Math. 37(2), 441-451 (2000)

19. Przytycki, J.H., Taniyama, K.: Almost positive links have negative signature. J. Knot Theory Ramif. 19(2), 187-289 (2010). arXiv:0904.4130 
20. Rudolph, L.: Algebraic functions and closed braids. Topology 22(2), 191-202 (1983). arXiv:math/0411316

21. Rudolph, L.: Constructions of quasipositive knots and links. III. A characterization of quasipositive Seifert surfaces. Topology 31(2), 231-237 (1992). arXiv:math/0411320

22. Rudolph, L.: Quasipositivity as an obstruction to sliceness. Bull. Am. Math. Soc. (N.S.) 29(1), 51-59 (1993). arXiv:math/9307233

23. Rudolph, L.: Quasipositive plumbing (constructions of quasipositive knots and links. V). Proc. Am. Math. Soc. 126(1), 257-267 (1998)

24. Rudolph, L.: Positive links are strongly quasipositive, Proceedings of the Kirbyfest (Berkeley, CA, 1998), Geom. Topol. Monogr., vol. 2, pp. 555-562. Geom. Topol. Publ., Coventry (1999). arXiv:math/9804003

25. Rudolph, L.: Quasipositive pretzels. Topol. Appl. 115(1), 115-123 (2001). arXiv:math/9908028

26. Stoimenow, A.: Knots of genus one or on the number of alternating knots of given genus. Proc. Am. Math. Soc. 129(7), 2141-2156 (2001)

27. Stoimenow, A.: On polynomials and surfaces of variously positive links. J. Eur. Math. Soc. 7(4), 477-509 (2005). arXiv:math/0202226

28. Stoimenow, A.: Knots of (canonical) genus two. Fund. Math. 200(1), 1-67 (2008). arXiv:math/0303012

29. Stoimenow, A.: Minimal genus of links and fibering of canonical surfaces. Ill. J. Math. 59(2), 399-448 (2015)

30. Stoimenow, A.: Knot data tables, retrieved 26 September. http://stoimenov.net/stoimeno/homepage/ ptab (2020)

31. Tagami, K.: The Rasmussen invariant, four-genus and three-genus of an almost positive knot are equal. Can. Math. Bull. 57(2), 431-438 (2014). arXiv:1411.2209v3

Publisher's Note Springer Nature remains neutral with regard to jurisdictional claims in published maps and institutional affiliations. 